\title{
1 Optimization of parabolic trough power plant operations in variable 2 irradiance conditions using all sky imagers
}

\author{
3 B. Nouri ${ }^{1}$, K. Noureldin ${ }^{2}$, T. Schlichting ${ }^{3}$, S. Wilbert ${ }^{1}$, T.Hirsch ${ }^{2}$, M. Schroedter-Homscheidt ${ }^{4}$, P. Kuhn ${ }^{1}$,
}

4 A. Kazantzidis ${ }^{5}$, L.F. Zarzalejo ${ }^{6}$, P. Blanc ${ }^{7}$, Z. Yasser ${ }^{8}$, J. Fernández ${ }^{9}$, R. Pitz-Paal ${ }^{10}$

${ }^{1}$ German Aerospace Center (DLR), Institute of Solar Research, Ctra de Senes s/n km 4, 04200 Tabernas, $6 \quad$ Spain

${ }^{2}$ German Aerospace Center (DLR), Institute of Solar Research, Wankelstrasse 5, 70563 Stuttgart, Germany

${ }^{3}$ German Aerospace Center (DLR), Institute of Solar Research, Solar Power Plant Technology, Prof.-RehmStr. 1, 52428 Jülich, Germany

${ }^{4}$ German Aerospace Center (DLR), Institute of Networked Energy Systems, Carl-von-Ossietzky-Straße 15, 26129 Oldenburg, Germany

${ }^{5}$ Laboratory of Atmospheric Physics, Department of Physics, University of Patras, 26500 Patras, Greece

${ }^{6}$ CIEMAT Energy Department - Renewable Energy Division, Av. Complutense 40, 28040 Madrid, Spain

${ }^{7}$ MINES ParisTech, 06904 Sophia Antipolis CEDEX, France

${ }^{8}$ TSK Flagsol Engineering GmbH, Anna-Schneider-Steig 10, 50678 Cologne, Germany

${ }^{9}$ CIEMAT-Plataforma Solar de Almería, Ctra. de Senés km 4.5, E-04200 Tabernas, Almería, Spain

${ }^{10} \mathrm{DLR}$, Institute of Solar Research, Linder Höhe, 51147 Cologne, Germany

\section{Abstract}

20 The continuously growing penetration of intermittent electricity sources will increase the future

21 demand for dispatchable power plants, which balance out fluctuations within the electrical grids.

22 Parabolic trough power plants with thermal energy storages could be one renewable solution for 23 regions with a high yearly direct normal irradiance (DNI) sum, but in order to compete against 
24 other renewable as well as non-renewable technologies, the economic competitiveness must be

25 ensured. Price reductions can be achieved for example by optimizing the plant operation. One

26 shortcoming of state of the art plant controllers is that they only use the DNI measured at one or

27 a few positions in the solar field. Due to the spatial variability of the DNI throughout the solar

28 field this DNI information can be misleading.

29 In this paper, we investigate the optimization potential of solar field control strategies with

30 access to spatially resolved DNI information from all sky imagers (ASI). Uncertainties of the ASI

31 system are considered by introducing additional independent spatial DNI information from a

32 shadow camera system. The spatial and temporal DNI variability of the DNI seen by the

33 controller is classified in distinct DNI variability classes. Two new control strategies are

34 developed, with optimized control parameters for distinct combinations of the spatial and

35 temporal DNI variability classes.

36 We observe significant improvements by these new variability class dependent control

37 strategies, compared to a state of the art reference controller. A relative increase in revenue in

38 excess of $1.9 \%$ is observed over a test period with 22 individual days.

39 Keywords

40 Parabolic trough, all sky imager, irradiance map, variability, solar field control, shadow camera, virtual solar field 


\section{Introduction}

43 The globally installed solar power capacity reached roughly 486 GW (IRENA 2019) by the end

44 of 2018 . Solar photovoltaic (PV) with about $480 \mathrm{GW}$ is the main contributor compared to $5.4 \mathrm{GW}$

45 for concentrating solar technologies (IRENA 2019). Currently, solar PV is also dominating the

46 overall global capacity increase, with additional 94 GW only in 2018 (IRENA 2019). Kost et al.

47 (2018) expects a strong continued growth of solar PV reaching a total global capacity between

$483000 \mathrm{GW}$ and $9000 \mathrm{GW}$ by the year 2035.

49 However, the strong growth of intermittent electricity sources, such as solar PV as well as wind

50 power, leads to new technological challenges. Fluctuations may cause unpredictable variations

51 of node voltages and power within the electrical grids, or even instabilities in case of

52 intermediate power shortages (Woyte et al. 2006; Perez et al. 2016). Especially small grids are

53 vulnerable to power ramps. Therefore, it comes as no surprise that Hawaii and Puerto Rico

54 already introduced legal limitations for ramp rates (Gevorgian \& Booth 2013; Crăciun et al.

55 2017). Larger grids with low penetration of intermittent sources can better compensate

56 fluctuations. But, in a case of a PV penetration above 15\% (annual energy basis), significant

57 changes in system operation are required also for large electrical grids (Denholm \& Margolis

58 2016).

59 For regions with high annual direct normal irradiance (DNI) sums, concentrating solar power

60 (CSP) plants with thermal energy storages (TES) provide a dispatchable and renewable energy

61 source (Platzer 2016). CSP is therefore capable of balancing fluctuations in electrical grids with

62 high penetration of intermittent sources (Mehos et al. 2016).

63 Yet, whether CSP is going to play an important role in the future global energy mix, is mainly a 64 question of costs. Significant reductions in levelized cost of electricity (LCoE) for CSP with 
65 storage were reached in the last couple of years (Lilliestam \& Pitz-Paal 2018), but the LCoE of

66 solar PV without storage remains significantly lower. However, CSP plants combined with TES

67 outcompete PV plants with battery storage of similar annual production and storage capacity,

68 especially for storage capabilities beyond 6 hours (Lilliestam et al. 2018). Such cost

69 comparisons should also take into account that CSP still offers a considerable cost reduction

70 potential. Price reductions can be achieved by scale effects, by improving the component

71 efficiencies or by optimizing the plant operation (Pitz-Paal 2017). This study contributes to the

72 latter objective.

73 The operation of commercial CSP power plants offers a lot of opportunities for optimization. In

74 the case of the most common CSP technology, parabolic troughs, collectors concentrate the

75 DNI on receiver tubes. A heat transfer fluid (HTF) circuits the receiver tubes and is heated up to

76 several hundred degrees Celsius. The thermal energy is passed over heat exchangers to a

77 conventional Rankine cycle or TES. Especially intra-hour and intra-minute DNI variability caused

78 by passing clouds pose an operational challenge for commercial parabolic trough power plants

79 with extensive solar field sizes (García et al. 2011; Hirsch et al. 2014). An efficient solar field

80 controller needs to find the best combination of field mass flow, temperature set-points and

81 defocusing of collectors corresponding to the prevailing DNI conditions (Wagner \& Wittmann

82 2014). Ideally, a perfect controller would adjust the mass flow in such a way, that the design

83 temperature is always maintained constant without any need of defocusing collectors. In order to

84 approach this ideal, the hydraulic and thermal interactions within the solar field must be well

85 understood - especially under transient conditions with a strong spatial and temporal variability

86 of the DNI inside the solar field. 
87 Comprehensive numerical models as developed by Hirsch \& Schenk 2010, García et al. 2011,

88 Giostri 2012, Zaversky et al. 2013, Noureldin et al. 2016 and Noureldin et al. 2017 are useful

89 to study the solar field behavior for distinct control strategies. The so called virtual solar field

90 (VSF), as presented and validated in Noureldin et al. 2016 and Noureldin et al. 2017,

91 simulates the entire solar field in a high temporal and spatial resolution and considers flow

92 maldistribution due to thermal transients and DNI inhomogeneity as reported e.g. in Abutayeh

93 et al. 2014. Furthermore, the VSF is designed to consider spatially inhomogeneous DNI

94 information, which occur during transient conditions and have a significant impact on the solar

95 field behavior.

96 Such spatially resolved DNI information can be provided by camera based monitoring and

97 nowcasting systems. These systems provide an intra-minute temporal resolution and a spatial

98 resolution $\leq 20 \mathrm{~m}$. The most common nowcasting systems consist of upward-facing all sky

99 imagers (ASI) (Chow et al. 2011; Quesada-Ruiz et al. 2014; Peng et al. 2015; Blanc et al.

100 2017; Kazantzidis et al. 2017; Nouri et al. 2019b). The principle method of these ASI systems

101 is most often similar. Images of the complete sky are taken and clouds are detected. If the

102 system consists of more than one ASI, the cloud height is determined via stereo photography or

103 similar approaches. Cloud shadows are projected to a ground model. The resulting shadow

104 maps are converted into irradiance maps via local irradiance measurements. For irradiance

105 predictions clouds can be tracked over consecutive sky images.

106 A less common and distinct nowcasting approach uses downward-facing cameras (Kuhn et al.

107 2017b). These so called shadow cameras are mounted on an elevated position and take images

108 of the ground. Shadow maps are created by detecting the cloud shadows within the ground

109 images. The shadow maps are converted into irradiance maps via local irradiance 
110 measurements and fully shaded as well as fully clear reference images taken for the same solar 111 position.

112 Noureldin et al. 2019a investigated the possible benefit of a parabolic trough solar field 113 controller with access to perfectly accurate spatial DNI information compared to a state of the art 114 controller with DNI information only from one or handful reference pyrheliometers distributed in 115 the solar field. Noureldin et al. 2019a utilizes the VSF and DNI maps from the ASI system 116 described by Nouri et al. 2019b. This initial study showed a significant potential with an 117 estimated gain in revenue up to $2.5 \%$ for some days with variable conditions. However, the 118 results presented in Noureldin et al. 2019a treat the DNI maps from the ASI system as perfectly 119 accurate descriptions of the ambient conditions. Yet, uncertainties exist, which also depend 120 strongly on the prevailing weather conditions (Marquez \& Coimbra 2013).

121 In this study, we will investigate the actual benefit of spatial DNI information from ASI systems 122 for a parabolic trough solar field controller with consideration of the system uncertainties. The 123 ASI derived DNI maps are provided to the solar field controller as DNI field average and a DNI 124 variability classification. The solar field controller is then adjusted according to the prevailing 125 spatial and temporal DNI variability class.

126 The uncertainties of the ASI system are considered by including additional spatial DNI 127 information from the fundamentally distinct shadow camera system (Kuhn et al. 2017b), which 128 provides the actual DNI conditions acting on the solar field, whereas the solar field controller will 129 receive only the DNI information from the ASI system (see Figure 1). Ideally, this allows the 130 quantification of the achievable impact of ASI based control schemes, with actual divergent 131 weather conditions unknown by the solar field controller. Of course, the shadow camera system 
132 is not perfect by itself but validation results illustrate that it outperforms the ASI systems and can 133 therefore be used as reference (see section 2.2).

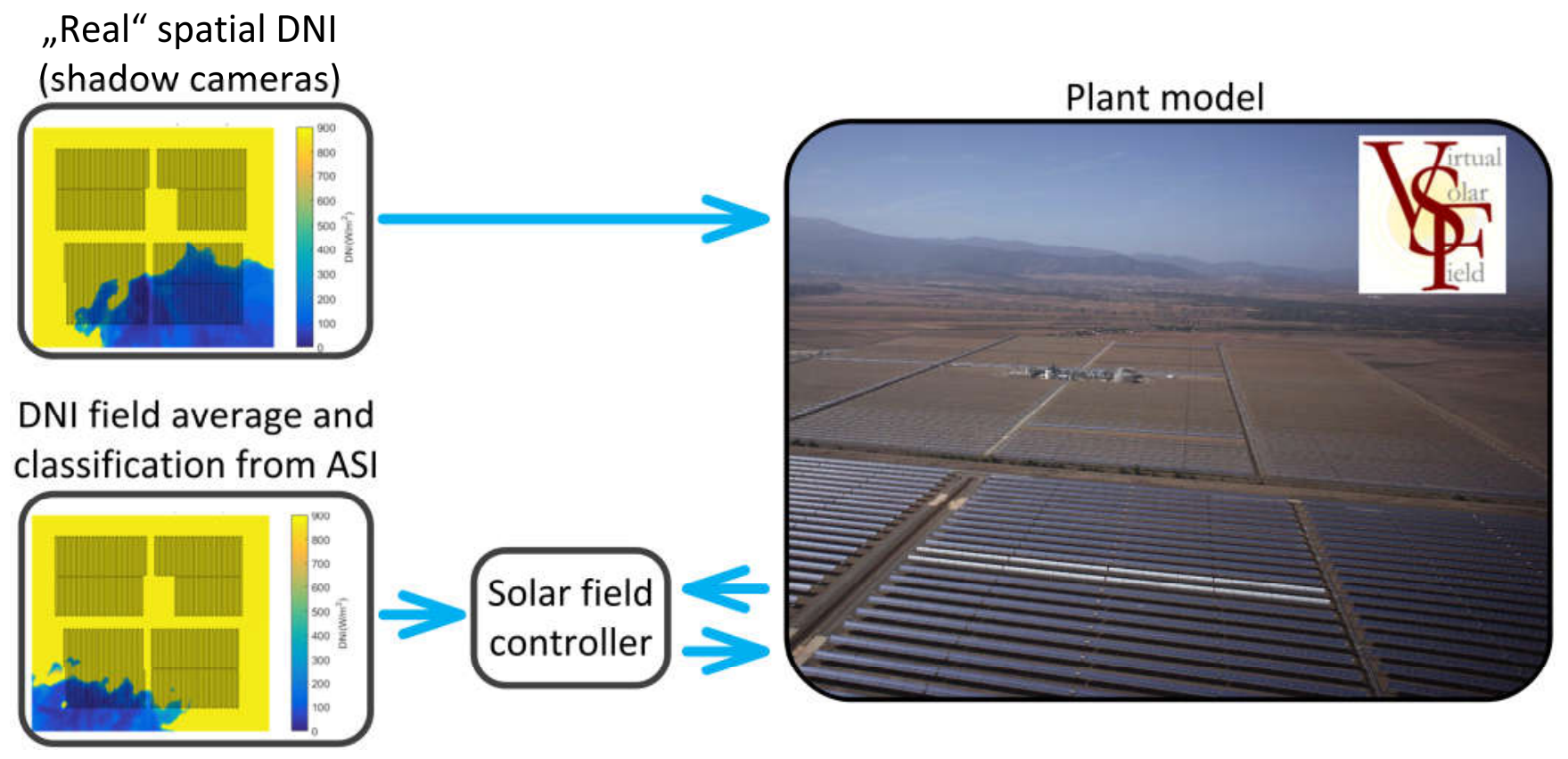

Figure 1: Schematic representation of the Virtual Solar Field (VSF) simulations with spatial DNI information from two distinct nowcasting systems

137 The ASI system also provides predictions up to 15 minutes ahead, which could be utilized by

138 model predictive controllers. Nevertheless, in this work we investigate only the potential benefit

139 of solar field control schemes with access to spatial DNI information of the current conditions.

140 All the presented evaluation strategies, including the ASI system and the VSF simulations, are

141 quasi real time capable with an average overall processing time of roughly 7 seconds per time 142 stamp (8x3.6GHz Intel Core i9-9900K, 2x16GB DDR4-2666 and PNY Quadro RTX 40008 GB

143 GDDR6). The fast processing time allows the solar field controller to obtain updates of the

144 irradiance situation in a timely manner.

145 Section 2 and section 3 of this paper will give an overview on the used ASI system and the VSF, 146 respectively. The developed temporal and spatial DNI variability classification method is 
147 presented in section 4. Two new solar field control strategies based on the DNI variability

148 classes are presented and evaluated versus a reference controller in section 5 . These new

149 controllers are tailored to a solar field design according to the Spanish commercial 50 MW

150 power plant La Africana. In section 6, we identify applicability conditions of the new variability

151 class dependent control strategies and present the performance results of a hybridized control 152 strategy. Finally section 7 concludes the paper.

1532 Overview on used camera systems that provide DNI maps

154 Two different sources for spatially and temporally resolved DNI information are used in this 155 study. Whereas the information of the ASI system is used as the available DNI information, 156 provides the shadow camera system the "real" DNI over the solar field. The following sections 157 introduce these two irradiance measuring devices.

\section{$2.1 \quad$ All sky imager system}

159 The used ASI system consists of two Mobotix Q24 of-the-shelf surveillance cameras and the 160 DNI information of a Kipp\&Zonen CHP1 pyrheliometer. The cameras are operated at CIEMAT's 161 Plataforma Solar de Almería (PSA) and mounted $494 \mathrm{~m}$ apart from each other. One of the 162 cameras stands at a distance less than $8 \mathrm{~m}$ away from the pyrheliometer. Q24 cameras are 163 equipped with fisheye lenses for taking hemispheric images of the sky. All sky images with a 3164 mega pixel resolution (see Figure 2) are taken simultaneously by both cameras every 30 165 seconds. 


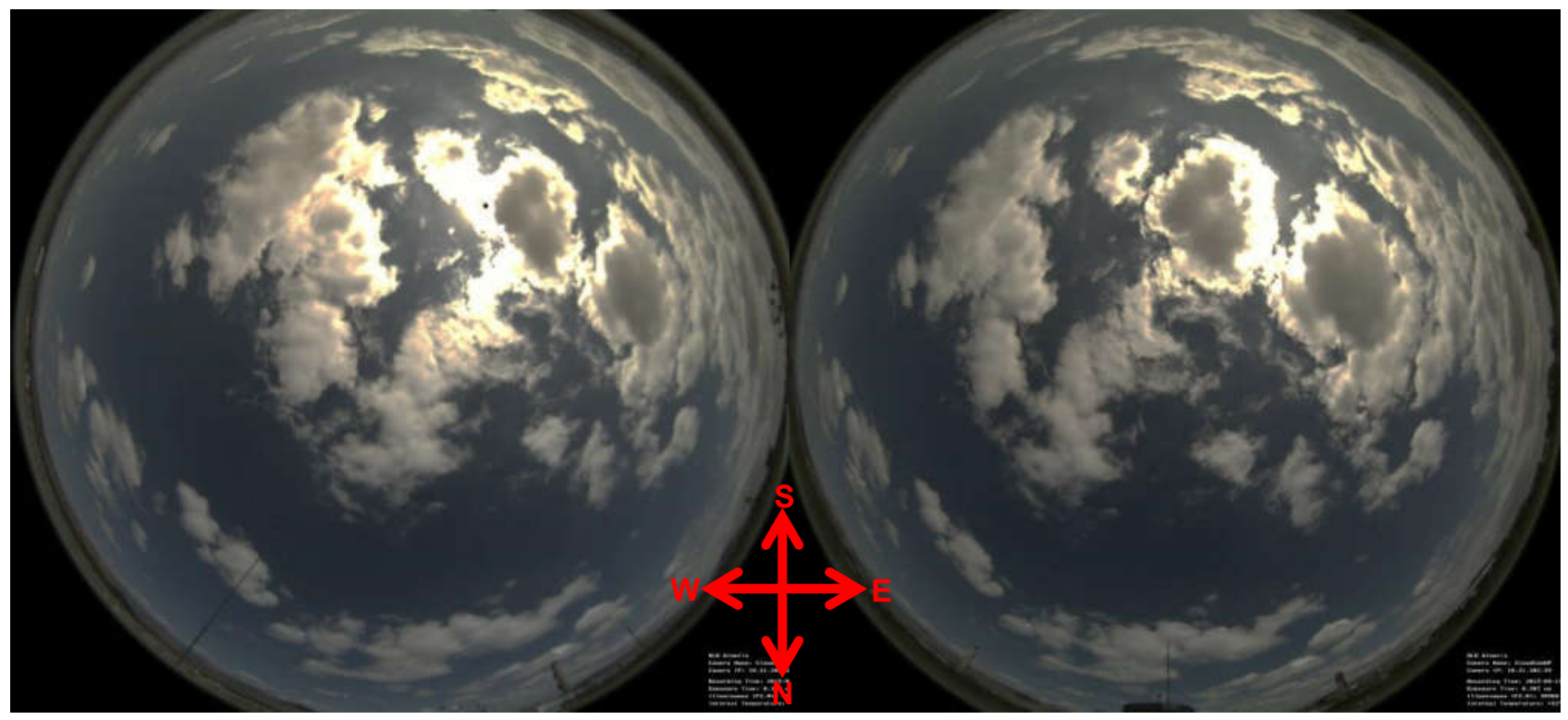

Figure 2: Example sky images as taken simultaneously by both Mobotix Q24 cameras

168 The image processing treats each detected cloud as an individual object with the attributes 169 position, motion, and transmittance as described in Nouri et al. 2018b. Clouds in the sky are 170 segmented by means of a four dimensional clear sky library (Wilbert et al. 2016; Kuhn et al. 171 2017a) which accounts for different atmospheric conditions. Geolocation and motion of the 172 clouds is detected by a stereoscopic approach based on differential images (Nouri et al. 173 2019a). A binary shadow map is created via a raytracing algorithm (Nouri et al. 2018b). A 174 probabilistic approach utilizes current and historical cloud transmittance and cloud height 175 measurements to allocate actual cloud transmittance values (Nouri et al. 2019b). Finally, a 176 binary shadow map is converted into a DNI map by introducing the current clear sky DNI and the 177 corresponding cloud transmittance values.

178 In the current configuration the DNI maps have an edge length up to $8 \mathrm{~km}$ with a spatial 179 resolution down to $5 \mathrm{~m}$. The overall ASI system and its sub systems were validated intensively. 180 Overall relative mean absolute deviation (MAD) of $4 \%$ and root mean square deviation (RMSD) 
181 of $7.8 \%$ are reached for the current conditions (lead time 0 minutes) over a two year period, 182 compared with three spatially distributed reference pyrheliometers (Nouri et al. 2019b).

\subsection{Shadow camera system}

184 ASI systems need to detect the position of clouds in the sky accurately in all three spatial 185 dimensions. Complex but common and frequently changing atmospheric conditions, partially 186 with multiple cloud layers, make this task challenging. A unique alternative to ASI systems are 187 shadow camera systems, which detect directly the cloud shadows on the ground, without the 188 detour over the clouds in the sky. The method and its validation is described in Kuhn et al. 189 2017b, possible applications are presented in Kuhn et al. 2019. At the Plataforma Solar de 190 Almeria (PSA) in Spain both the ASI system as described in section 2.1 and the shadow camera 191 system are operated jointly. The shadow camera system consists of six Mobotix M24 off-the192 shelf surveillance cameras mounted on the top of a $87 \mathrm{~m}$ high solar tower. Figure 3 shows one 193 of the shadow cameras and an example image as taken from the tower looking to the ground.

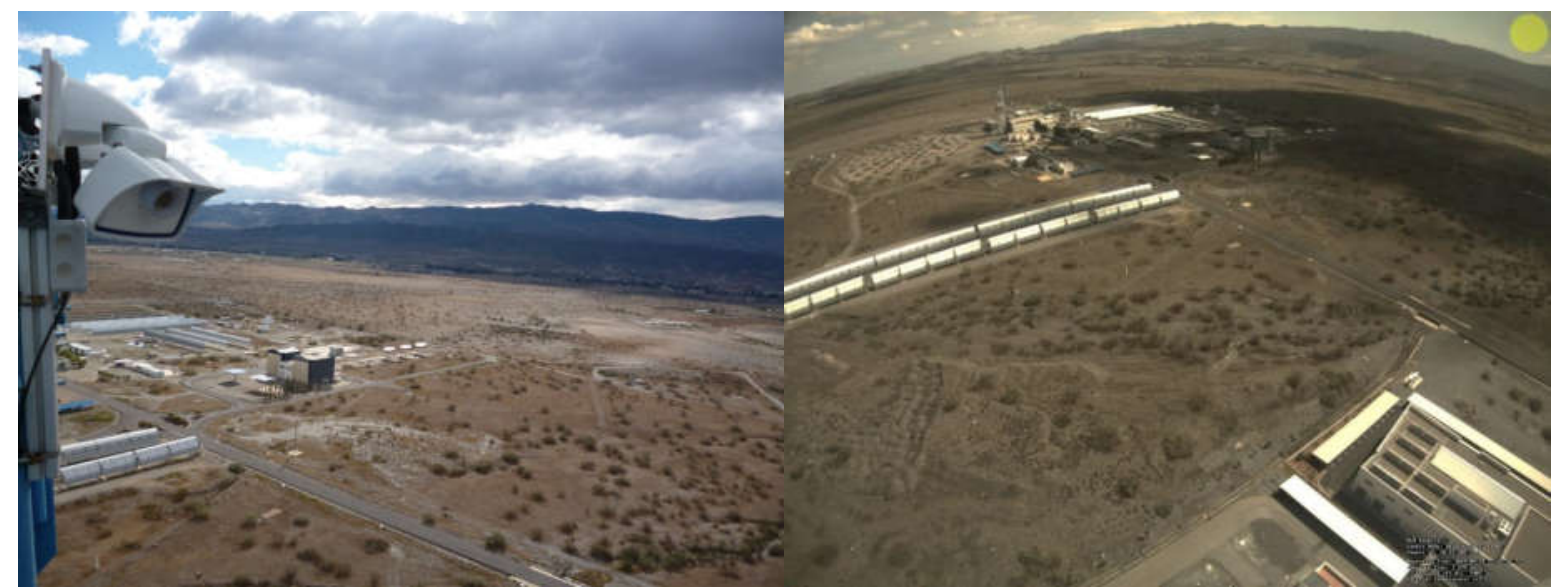

Figure 3: (left) Shadow camera mounted on top of a solar tower (right) image of a shadow camera

196 The viewing cones of the six cameras cover a $360^{\circ}$ view around the tower. All cameras take 197 simultaneously every 15 seconds a new image of the ground which are converted to a single 198 orthoimage (Figure 4). 


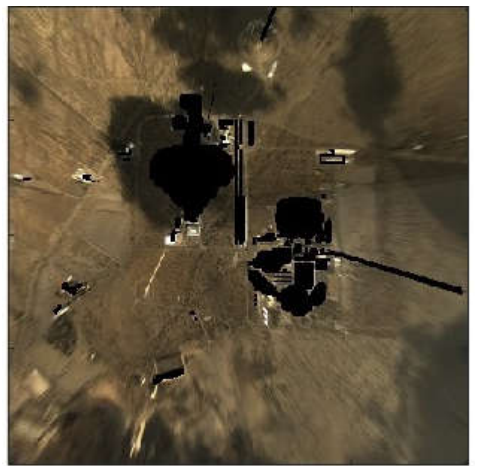

200 Figure 4: Orthoimage with a $360^{\circ}$ view created from images of six shadow cameras mounted on the top of a solar tower.

201 A difference image is calculated between the actual orthoimage and an additional reference

202 clear sky orthoimage from a data base corresponding to a similar Sun position with less than $3^{\circ}$

203 deviation of azimuth and elevation angle. Furthermore, the reference image must be taken less

204 than 60 days before the current image, which avoids significant differences in ground properties.

205 Shadows on the ground are segmented by comparing each pixel value of the difference image

206 with empirically identified thresholds. The shadow camera system uses the DNI measurements

207 taken by a pyrheliometer and DHI measurements taken by a pyranometer with a shadow ball.

208 Unshaded sections of the orthoimage receive the clear sky DNI values identified by the most

209 recent clear DNI measurements detected according to Hanrieder et al. 2016. For the

210 identification of the DNI within the shaded sections a second reference orthoimage image for

211 overcast conditions is needed. The second reference image must be taken in the last 60 days

212 and within $10^{\circ}$ of the Sun elevation and azimuth angle for the evaluated image. The DNI in the

213 shaded pixels is calculated according to Kuhn et al. 2017b from the rgb values of the three

214 orthoimages and the DHI measurement. The sunny and the shaded reference image are used to

215 approximate the bidirectional reflectance distribution function of each pixel in the image for the

216 current solar position and the position of the camera. The final DNI maps have a spatial

217 resolution of $5 \mathrm{~m}$ with a maximum edge length of $2 \mathrm{~km}$. 
218 A benchmarking campaign between the ASI system described in section 2.1 and the shadow 219 camera system with three reference pyrheliometer over 22 variable days was conducted by 220 Kuhn et al. 2019. For lead time 0 minutes, the shadow camera system reached a rel. RMSD of $22110.2 \%$, rel. MAD of $6.7 \%$ and a rel. bias of $3.3 \%$ compared to a rel. RMSD of $15.1 \%$, rel. MAD of $2229.2 \%$ and a rel. bias of $6.7 \%$ for the ASI system.

\section{Virtual Solar Field}

\subsection{Overview of virtual solar field}

225 Parabolic trough solar fields of commercial power plants are spatially extended facilities with 226 edge lengths typically above $1 \mathrm{~km}$. The solar field is divided into several sections, whereas each 227 section consists of a multitude of parallel loops. Each loop itself consists of several solar 228 collector assemblies (SCA), which describe the smallest collector unit with an independent drive 229 for tracking. The incoming DNI is concentrated by parabolic shaped collectors to the focal line. 230 Receiver tubes are located in the focal line. A heat transfer fluid circulates through the receiver 231 tubes. All loops of the same section are connected to a cold and a hot section header pipe. In 232 turn, all the section header pipes are connected to a cold or hot main header pipe, which 233 connects the solar field sections to the power block. A schematic solar field layout is illustrated in

234 Figure 5. The control of such a solar field is a complex hydraulic as well as a thermal challenge. 235 The loops of the solar field are not equipped with individual flow control valves. This means that 236 the flow through the field is only determined by the power of the main pump supplying the whole 237 solar field. The flow distribution over the sectors and loops is thus defined by the hydraulic 238 resistance of each of the parallel loops in the network. 


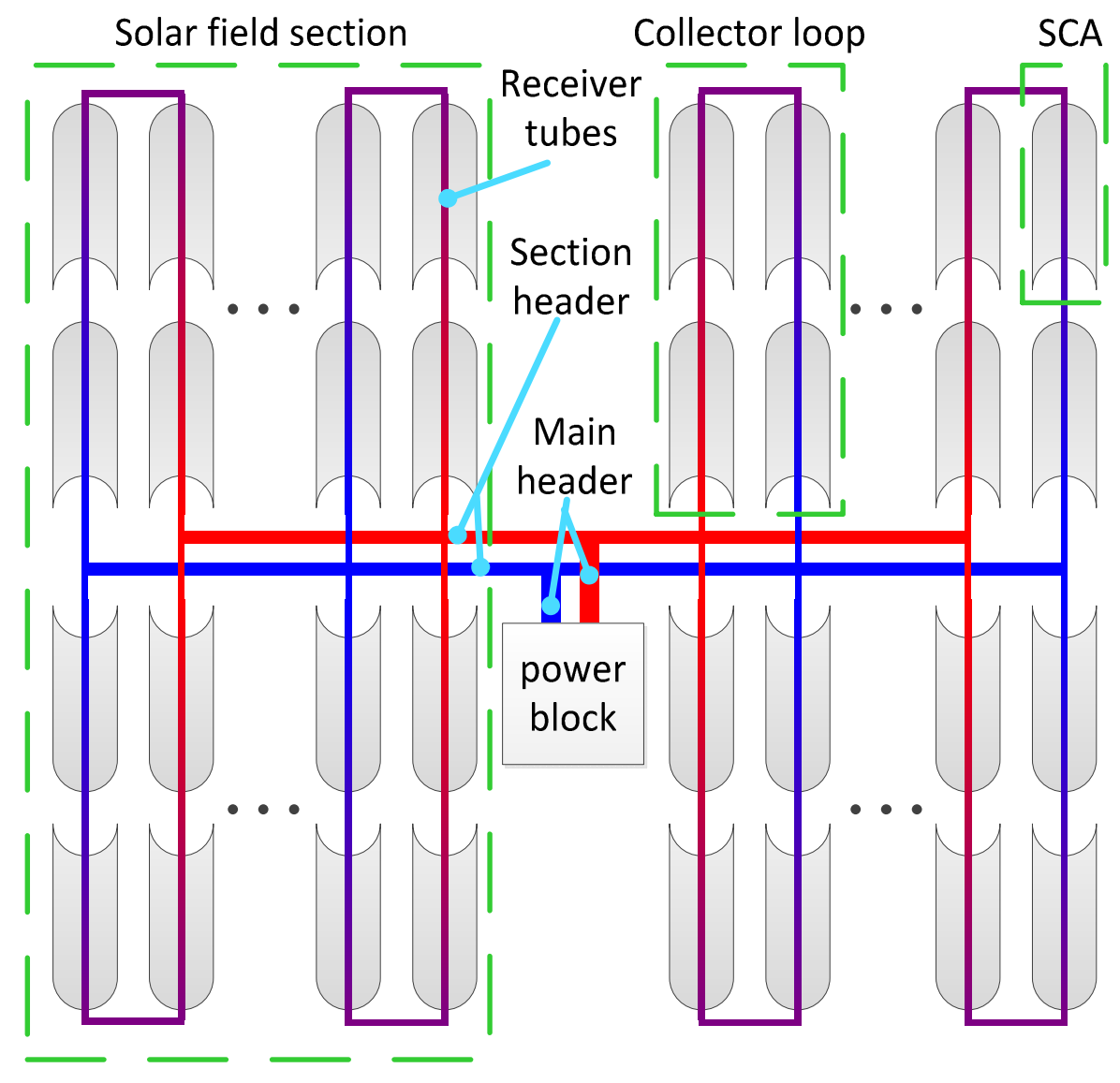

Figure 5: Schematic solar field layout

241 The VSF is a simulation environment which models the entire solar field from the power block.

242 Common solar field designs with an arbitrary number of loops can be realized with the VSF

243 software. In order to accurately predict the physical behavior of the solar field, VSF couples a

244 hydraulic network solver with a thermal solver. A detailed description of VSF can be found in

245 Noureldin et al. 2016 and Noureldin et al. 2017. The VSF principles and the existing validation

246 of the VSF are summarized below.

247 The hydraulic solver computes the flow distribution within the branched piping system based on

248 steady state assumptions. For this purpose, the solar field is discretized in pipe elements of

$24912.5 \mathrm{~m}$ length within the collectors and $15 \mathrm{~m}$ to $50 \mathrm{~m}$ length within the header piping. Due to 250 strong variations in temperature within the solar field (also during stable conditions), the 
251 hydraulic resistance is computed for each discrete element with a temporal resolution of 2

252 seconds. Therefore, VSF is also capable of characterizing flow maldistribution due to thermal

253 transients as e.g. triggered by variable solar irradiance conditions or fluid distributions due to

254 inhomogeneities in plant design or operations.

255 The hydraulic solver passes flow boundary conditions to the thermal solver, which in turn

256 dynamically computes the temperatures with respect to the local thermal and operation

257 conditions, and the thermal losses within each discrete pipe element. These calculations solve

258 the time-dependent continuity and energy equations based on the assumption of a one

259 dimensional flow. Previously published empirical relations are utilized for the thermal losses (e.g.

260 Burkholder and Kutscher 2009 for Schott PTR-70 receiver tubes). At pipe intersections the 261 temperatures are computed by enthalpy balancing.

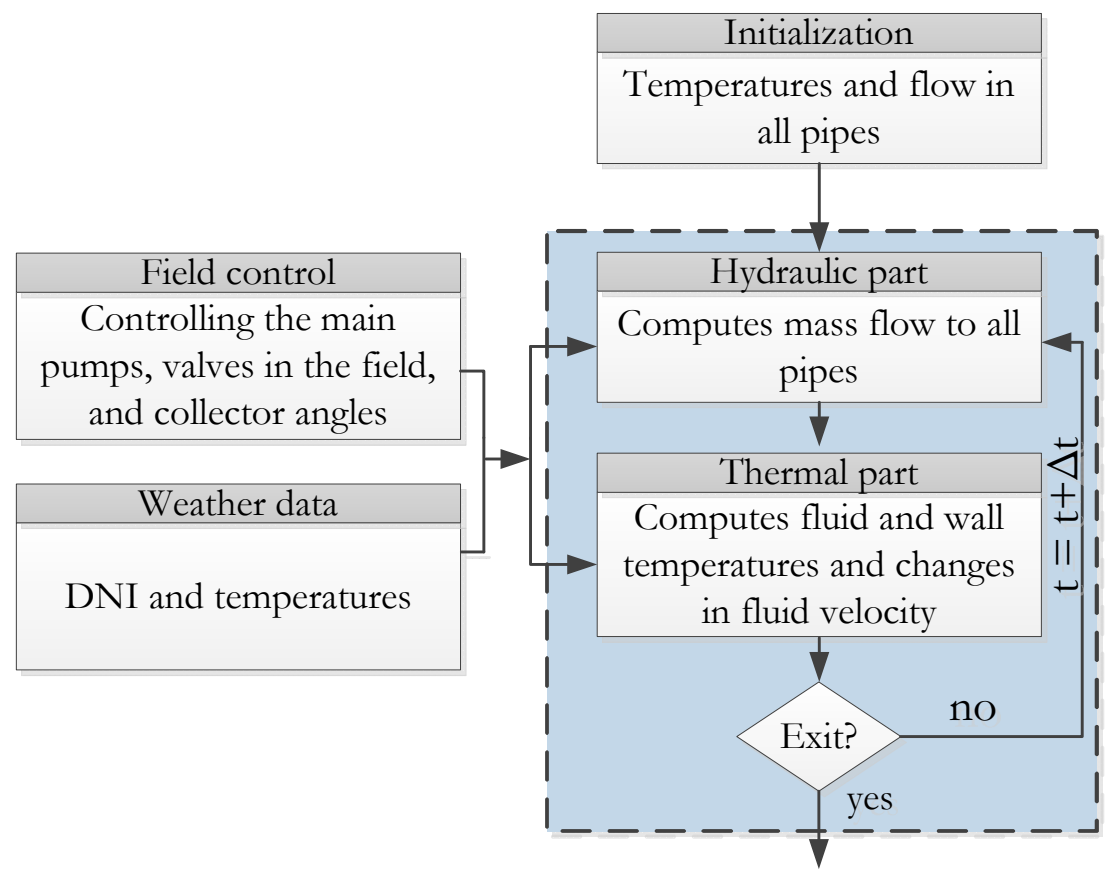

Figure 6: Illustration of the VSF program flow (adapted from Noureldin et al. 2018a)

264 A comprehensive validation of VSF was conducted based on a few days in Spring 2015 and real 
266 southern Spain (Noureldin et al. 2017). This power plant consists of four subfields with 38 loops

267 each. The power plant data were supported by additionally measured values from a

268 measurement campaign carried out simultaneously by the DLR at Andasol 3 on three test loops

269 (Noureldin et al. 2016). These additional data provide flow and temperature measurements

270 within the test loops with a high temporal resolution. For this purpose the loops were equipped

271 with a FLEXIM FLUXUS ADM 6725 clamp on ultrasonic flow meters with wave injectors for high

272 temperature applications at the loop center (cross over piping) and a clamp-on temperature

273 measuring system based on PT100s at the loop inlet and outlet (Nouri et al. 2018a). The

274 validation was conducted over distinct cases including normal operations with predominantly

275 clear conditions, start-up situations in clear mornings and conditions with strong transients due

276 to cloud passages. For the flow distribution, deviations below $5 \%$ between the simulated and

277 measured values were observed. During normal operations an overall RMSD in fluid

278 temperature of $2.75 \mathrm{~K}$ is observed. The RMSD rises up to $9.7 \mathrm{~K}$ during strong defocusing cycles.

279 These deviations are not significant compared to the expected uncertainties of the power plant 280 instrumentation (Janotte 2012).

\subsection{Solar field controller assessment with the VSF}

282 VSF is a useful tool to comparatively assess distinct solar field controllers in terms of the yield.

283 In Noureldin et al. 2018b and Noureldin et al. 2019a an approach is presented, which allows a 284 monetary assessment of parabolic trough solar field controller with the results of VSF 285 simulations. The solar field control concept is described in section 5 . We will roughly summarize 286 the evaluation approach here, for the better understanding of the results presented in sections 5 287 and 6 . Since the mass flow can only be altered for the whole field but not for each individual 288 loop, inhomogeneous irradiance conditions or flow distribution may cause some loops to 289 temporarily overheat while others do not reach the set point temperature. A methodology based 
290 on economic penalties has been developed to assess the solar field controller quality. The total 291 economic penalty is composed as

$p_{\text {tot }}=p_{\text {def }}+p_{T, P B}+p_{T E S} \cdot$

Equation 1

292 The first term $p_{\text {def }}$ describes a loss of revenue due to defocused collectors where the amount of

293 not usable heat $Q_{d e f}$ is weighted with the power block $\eta_{P B, a}$ efficiency and levelized cost of

294 electricity $L C o E$,

$p_{d e f}=Q_{d e f} \cdot \eta_{P B, a} \cdot L C o E$

Equation 2

295 The second effect is given by the fact that any reduction in solar field outlet temperature leads to 296 a reduction of the power block efficiency. The overall heat produced by the solar field is again 297 weighted with the LCoE and the averaged power block efficiency corrected by temperature 298 effects. The reduced power block efficiency penalty $p_{T, P B}$ is described by

$p_{T, P B}=Q_{t h, S F} \cdot \eta_{P B, a} \cdot L C o E \cdot\left(1-\frac{\eta_{P B, T}}{\eta_{P B, T_{0}}}\right)$

Equation 3

299 with $Q_{t h, S F}$ as the thermal solar field energy, $\eta_{P B, T_{0}}$ the power block efficiency at the design

300 temperature and $\eta_{P B, T}$ as the power block efficiency at the current temperature.

301 Reduced solar field temperatures have also an effect on TES efficiency. This is due to an overall 302 lower average temperature within the hot tank of the TES. This effect can be described by the 303 TES penalty

$p_{T E S}=Q_{t h, S F} \cdot \eta_{P B, a} \cdot L C o E \cdot\left(1-\frac{T_{o u t}-T_{i n}}{T_{0}-T_{\text {in }}}\right) \cdot \frac{Q_{O L, a}}{Q_{T E S, a}}$ Equation 4 
304 with $T_{\text {out }}$ as the solar field outlet temperature, $T_{\text {in }}$ as the solar field inlet temperature, $T_{0}$ as the 305 design temperature for the solar field outlet and $\frac{Q_{O L, a}}{Q_{T E S, a}}$ as the annually averaged ratio of TES 306 overload resulting in solar energy dumping.

307 The quantities $\eta_{P B, a}, L C O E$ and $\frac{Q_{O L, a}}{Q_{T E S, a}}$ are determined as average values over one year by means 308 of annual yield simulations conducted with the Greenius simulation software (Dersch et al. 309 2012; Dieckmann 2017). For the La Africana power plant considered in sections 5 and 6, the 310 following values are used $\eta_{P B, a}=0.25, L C O E=192.5 \frac{€}{M W h}$ and $\frac{Q_{O L, a}}{Q_{T E S, a}}=0.33$. The theoretical 311 revenue $R_{t h}$ and the actual revenue $R_{S F}$ are calculated according to the Equation 5 and Equation 3126.

$$
\begin{aligned}
& R_{t h}=\eta_{P B, a} \cdot L C o E \cdot \sum_{S C A} \int\left[G_{e f f}(t) \cdot \eta_{o p t} \cdot A_{a p}\right]_{S C A} d t \\
& R_{S F}=\eta_{P B, a} \cdot L C o E \cdot \int \dot{Q}_{t h, S F} d t-\left(p_{T E S}+p_{T, P B}\right)
\end{aligned}
$$

313 Thermal losses in the field are computed as

$$
Q_{\text {loss }, o t h}=R_{t h}-p_{\text {def }}-\eta_{P B, a} \cdot L C o E \cdot \int \dot{Q}_{t h, S F} d t
$$

314 with $G_{\text {eff }}$ as the effective solar irradiance falling on the collector, $\eta_{\text {opt }}$ as the optical collector 315 efficiency and $A_{a p}$ as the collector aperture area. 


\section{DNI variability classes}

\subsection{Temporal variability classes}

318 Temporal DNI variability classes are introduced to categorize irradiance situations based on the

319 prevailing variability in the solar resource. They can be used for systematic analysis and, as in

320 this study, for selecting situation dependent controllers.

321 The temporal DNI variability classification is determined according to the procedure described in 322 Schroedter-Homscheidt et al. 2018 with the adaptations described in Nouri et al. 2019c. This 323 approach analyses 13 distinct variability indices and classifies the DNI in one of eight classes

324 form clear sky (class 1 ) to overcast (class 8). The definition of the distinct classes is given in 325 Table 1. Each time stamp is classified by analyzing the DNI information of the previous 15 326 minutes (1 minute average DNI values).

Table 1: Description temporal DNI variability classes

\begin{tabular}{|c|c|c|c|}
\hline Class & Variability & $\begin{array}{c}\text { Clear sky index } \\
\left(D N I / D N I_{\text {clear }}\right)\end{array}$ & General description \\
\hline 1 & Low & Very high & Clear sky \\
\hline 2 & Low & High & Almost clear sky \\
\hline 3 & Intermediate & High/Intermediate & Almost clear sky \\
\hline 4 & High & Intermediate & Partly cloudy \\
\hline 5 & Intermediate & Intermediate & Partly cloudy \\
\hline 6 & High & Intermediate/Low & Partly cloudy \\
\hline 7 & Intermediate & Low & Almost overcast \\
\hline 8 & Low & Very Low & Overcast \\
\hline
\end{tabular}

329 As an example we show in Figure 7 the DNI, clear sky DNI and the corresponding temporal DNI 330 variability class for the 09.09 .2015 (PSA). The forenoon of this day is mainly dominated by clear 
331 sky conditions. More variable conditions start shortly before 11 UTC. The afternoon shows high

332 and intermediate variability conditions mainly dominated by class 5 .

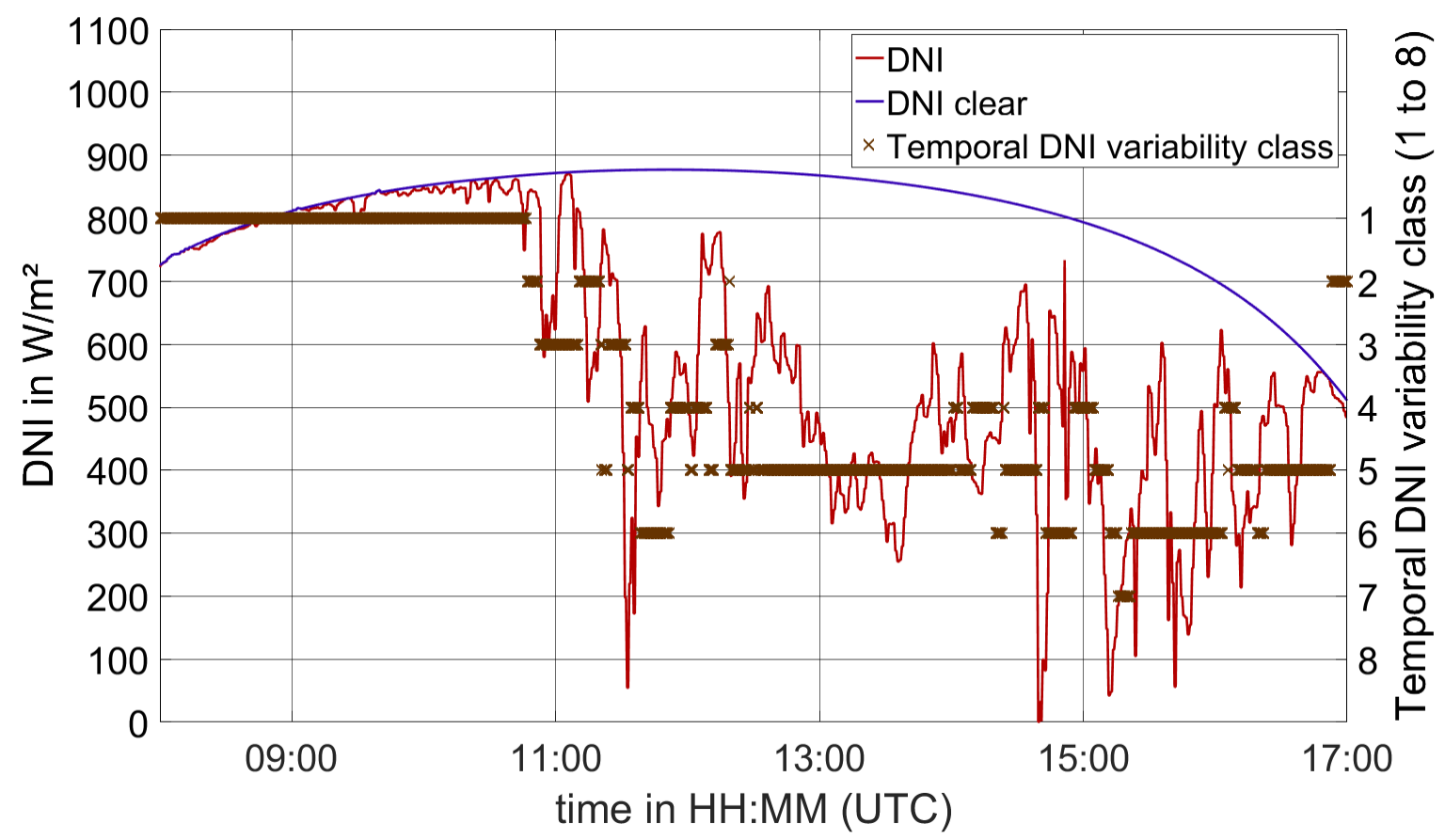

Figure 7: Temporal DNI variability classes of an example day (09.09.2015)

\subsection{Spatial variability classes}

336 The temporal variability classification is a method to describe the temporal variability as

337 observed in a ground observation, but it does not describe the spatial pattern involved in detail.

338 The impact of spatial heterogeneity of DNI on the solar field can be severe even if the temporal 339 variability is low. For example, conditions could occur where only half of the solar field is shaded 340 by clouds with a low transmittance while the other half is fully illuminated. From the viewpoint of 341 temporal variability, the situation is not variable at all if the cloud is not moving horizontally, or it 342 presents a low temporal variability if the cloud edge moves across the ground observation 343 pinpoint. 
344 In such a case, the two pyrheliometers used for a control may be placed one in the shaded and 345 one in the sunny area. The controller will adjust the flow of the HTF according to the average 346 solar field DNI. This leads to a partial cooling and overheating of the solar field which in turn 347 leads to a flow maldistribution. An even stronger imbalance between shaded and sunny shares 348 of the solar field could increase this issue - if not known to the controller. Especially problematic 349 are profound overheating issues within sunny parts of the solar field, causing defocusing and 350 energy dumping. The share between shaded and unshaded parts of the solar field is therefore a 351 decisive value for the solar field behavior and requires a specific treatment. Therefore, two new 352 variability indices derived from spatial DNI maps are introduced.

353 - The shadow area fraction $S_{A}$ describes the relative shaded share of the solar field $\circ S_{A}=\frac{A_{S A S F}}{A_{S F}} \cdot 100$, where $\mathrm{A}_{\mathrm{SASF}}$ is the shaded solar field area and $\mathrm{A}_{\mathrm{SF}}$ is the complete solar field area.

- Furthermore, the shaded clear sky index $S_{i}$ describes the clear sky index considering only the shaded part of the solar field

○ $S_{i}=\frac{D N I_{S A S F}}{D N I_{\text {clear }}}$, where $\mathrm{DNI} \mathrm{SASF}_{\text {is }}$ is the average DNI within shaded areas of the solar field and $\mathrm{DNI}_{\text {clear }}$ is the clear sky DNI.

360 Average values of the spatial DNI variability indices from the last 5 minutes are calculated. The 361 classification in one of 5 spatial DNI variability classes is done according to the thresholds given 362 in Table 2. Figure 8 illustrates a partially shaded parabolic trough solar field and the 363 corresponding instantaneous DNI variability indices. 
Table 2: Thresholds for spatial variability classes

\begin{tabular}{|c|c|c|c|}
\hline Class & $\begin{array}{l}\text { shadow area fraction } S_{A} \\
{[\%]}\end{array}$ & $\begin{array}{l}\text { Shaded clear sky index } \\
S_{i}\end{array}$ & General description \\
\hline 1 & $0-3$ & no condition & Sunny conditions \\
\hline 2 & $3-100$ & $0.9-1.0$ & $\begin{array}{l}\text { Low spatial DNI variability: } \\
\text { Only shadows from clouds with high transmittance }\end{array}$ \\
\hline 3 & $3-50$ & $0-0.9$ & High spatial DNI variability \\
\hline 4 & $50-100$ & $0.35-0.9$ & $\begin{array}{l}\text { Intermediate spatial variability: } \\
\text { Most of the solar field shaded } \\
\text { Cases with only thin clouds and only thick clouds } \\
\text { excluded }\end{array}$ \\
\hline 5 & $60-100$ & $0-0.35$ & $\begin{array}{l}\text { Low spatial variability: } \\
\text { Most of the solar field shaded (overcast) } \\
\text { Only shadows from clouds with low transmittance }\end{array}$ \\
\hline
\end{tabular}

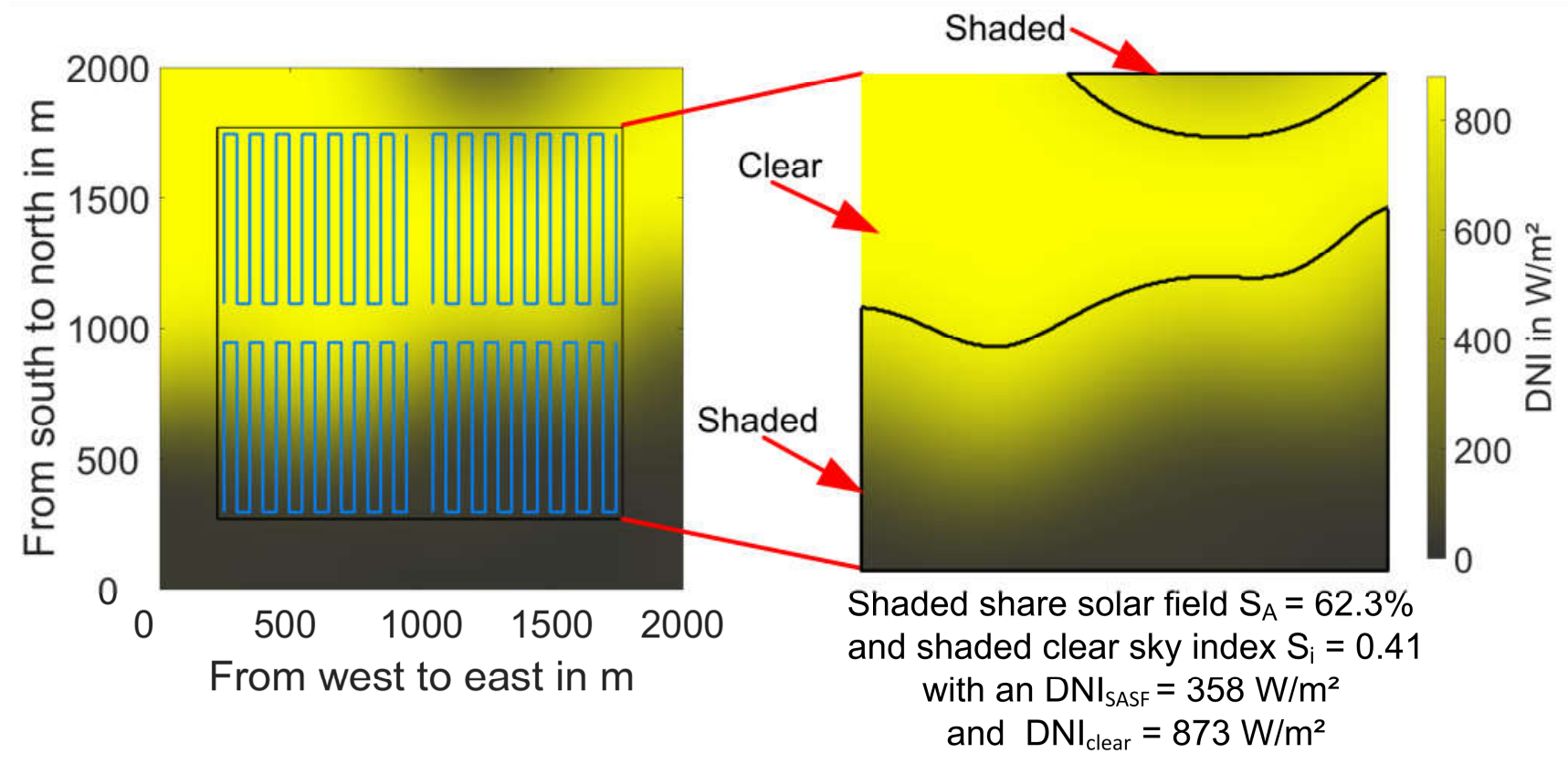

Figure 8: Partially shaded solar field with a shaded share of $62.3 \%$ and a clear sky index of 0.41 corresponding to spatial DNI variability class 4

371 We use again the 09.09.2015 (PSA) as showcase (Figure 9). Similar to the temporal DNI

372 variability classification, the forenoon is classified as sunny (class 1 ), whereas the more variable

373 afternoon shows a stronger diversification with changing classes dominated by class 4. 


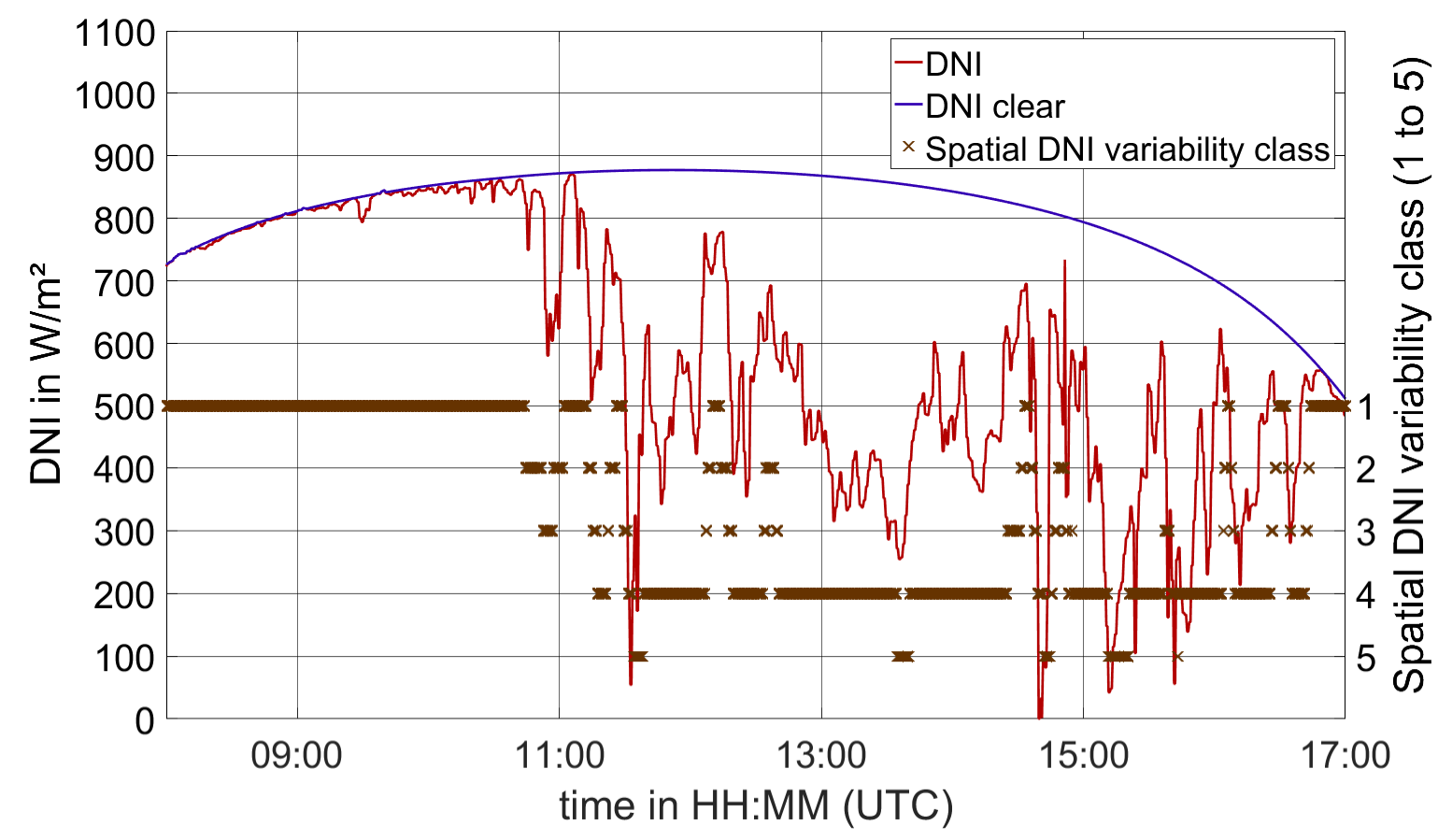

Figure 9: Spatial DNI variability classes of an example day (09.09.2015)

\subsection{Combined temporal and spatial variability classes}

377 The temporal and spatial DNI variability classes describe two distinct classification approaches

378 of the DNI with different emphases. Theoretically, 40 combinations of temporal and spatial DNI

379 variability exist, but in realistic cloud situations not all combinations are likely to occur. The

380 distribution of all occurred combinations for two complete years at the PSA is depicted in Figure

381 10. The most common conditions for the PSA are described by a spatial DNI variability class 1

382 and a temporal DNI variability class 1 or 2 . These clear sky conditions account for more than

$38362 \%$ of the entire data set.

384 Around $13 \%$ of the data set consists of intermediate to highly variable conditions both in time 385 and space (spatial class 3 or 4 and temporal class 3 to 7 ), which are the most demanding 386 situations for the parabolic trough plant control. From the view of the parabolic trough plant 387 control less important overcast conditions occur in $14.8 \%$ of the data set (spatial class 5 and 388 temporal class 6 to 8 ). Roughly $4 \%$ of the data set is described by a spatial class 2 and temporal 
389 class 1 to 7 . Such conditions with a low spatial variability but alternating temporal variability 390 occur mainly due to optical thin cirrus clouds, covering the whole power plant area as a single 391 cloud object, but having an internal spatial heterogeneity in the cloud optical depth. The 392 remaining conditions describe mainly transient conditions with an average occurrence of $0.1 \%$ 393 per combination of spatial and temporal class. Two very extreme examples are spatial class 5 394 and temporal class 1 as well as spatial class 1 and temporal class 7 , with an occurrence of $3950.29 \%$ and $<0.01 \%$, respectively. The conditions at PSA are quite attractive for a parabolic 396 trough power plant and thus relevant for the investigations carried out in this work. For the year 3972017 the yearly DNI sum at PSA was $2430 \mathrm{kWh} / \mathrm{m}^{2}$.

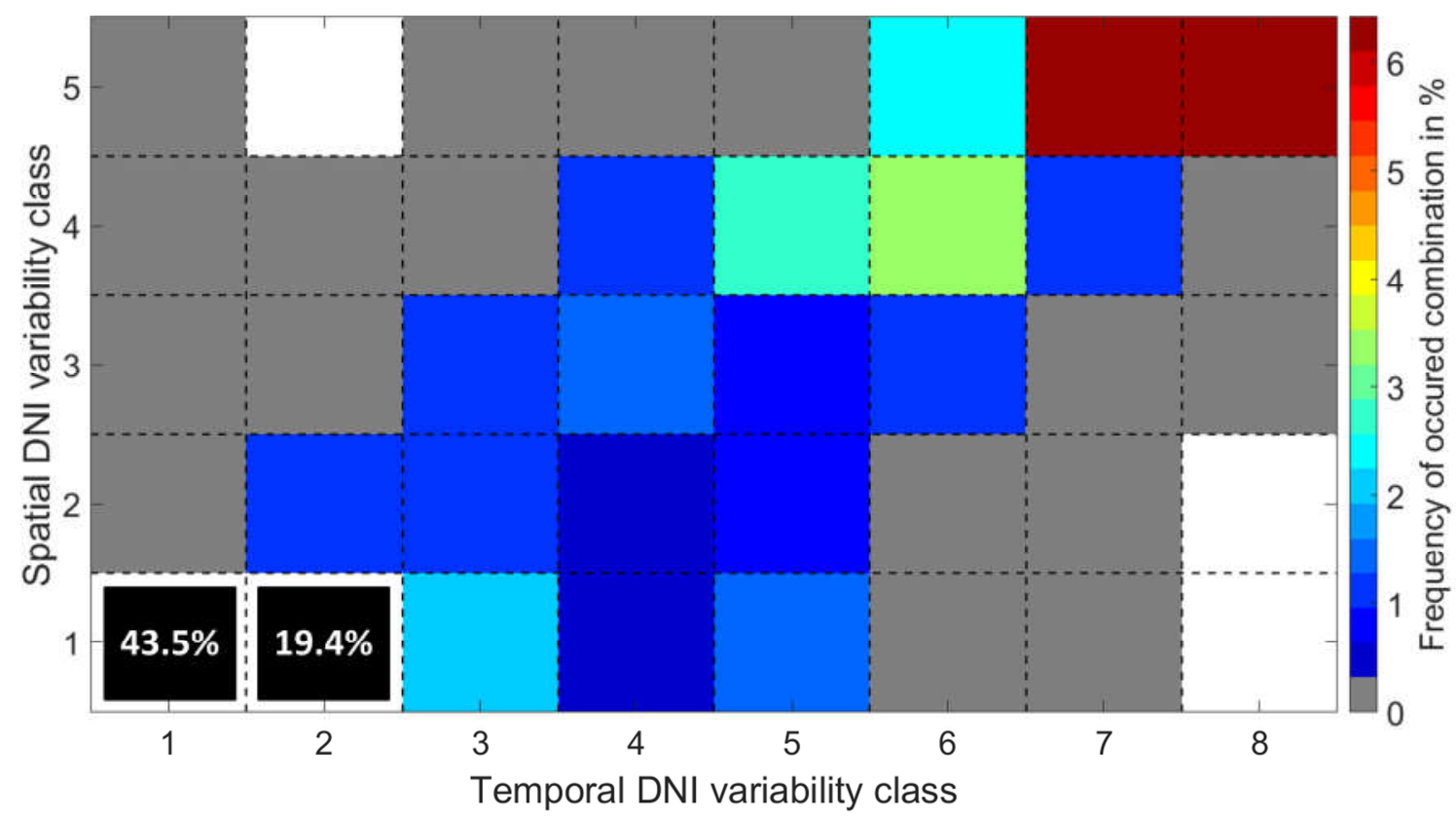

Figure 10: Temporal and spatial DNI variability class distribution of possible combinations within the years 2016 and 2017. Combinations of spatial class $1 \&$ temporal classes 1 or 2 account for more than $62 \%$ of the data set. These bins are not described by the colorbar for a better readability of the remaining combinations.

402 Based on this assessment, the 40 possible combinations of spatial and temporal DNI variability 403 classes are combined to 7 aggregated classes according to Table 3. Due to the power plants 404 strong sensitivity on the spatial solar share, the main focus is set on the spatial variability, which 
405 describes the distribution of the DNI over the spatially extended solar field. In cases of e.g.

406 spatial class 1 , the temporal variability classification is not decisive, as all temporal classes are

407 attributed to the combined variability class 1 . The temporal variability classification is relevant in 408 conditions with a strong spatial as well as temporal variability - and therefore treated separately 409 with the combined classes 6 and 7 ,

Table 3: Combined temporal and spatial variability classes

\begin{tabular}{|c|c|c|l|}
\hline $\begin{array}{l}\text { Combined } \\
\text { variability class }\end{array}$ & $\begin{array}{l}\text { Spatial } \\
\text { variability } \\
\text { class }\end{array}$ & $\begin{array}{l}\text { Temporal } \\
\text { variability } \\
\text { class }\end{array}$ & General description \\
\hline 1 & 1 & $1-8$ & $\begin{array}{l}\text { Sunny conditions with no spatial variability; all temporal } \\
\text { variability classes are accepted }\end{array}$ \\
\hline 2 & 3 & $1-3,5-8$ & $\begin{array}{l}\text { Low spatial DNI variability (almost completely sunny solar field); } \\
\text { total spectrum of temporal variability possible }\end{array}$ \\
\hline 3 & 4 & $1-3,5,7-8$ & $\begin{array}{l}\text { High spatial DNI variability; Almost complete spectrum of } \\
\text { temporal variability possible except highly temporal variable } \\
\text { class 4 with an intermediate clear sky index }\end{array}$ \\
\hline 4 & 5 & $1-8$ & $\begin{array}{l}\text { Intermediate spatial variability; only low and intermediate } \\
\text { temporal variability }\end{array}$ \\
\hline 5 & 3 & 4 & $\begin{array}{l}\text { Current overcast conditions with no spatial variability; total } \\
\text { spectrum of temporal variability possible }\end{array}$ \\
\hline 6 & 4 & 4 or 6 & $\begin{array}{l}\text { Intermediate spatial variability; only highly temporal variable } \\
\text { classes 4 and 6 }\end{array}$ \\
\hline 7 & 2 & 1 intermediate clear sky index \\
\hline
\end{tabular}

411 Looking again into our example day the 09.09.2015 (see Figure 11), we see a stronger

412 diversification within the variable time window from 10:45 to 16:45 compared to the previous

413 individual variability classifications. Whereas the purely spatial classification within this period is

414 dominated by class 4 with a share of $65 \%$ (see Figure 9 ), the combined class is dominated by

415 the classes 4 and 7 with a share of $36 \%$ and $29 \%$, respectively. Both the combined classes 4

416 and 7 represent an overall intermediate spatial variability, but class 7 represents in addition a

417 high variability in time. 


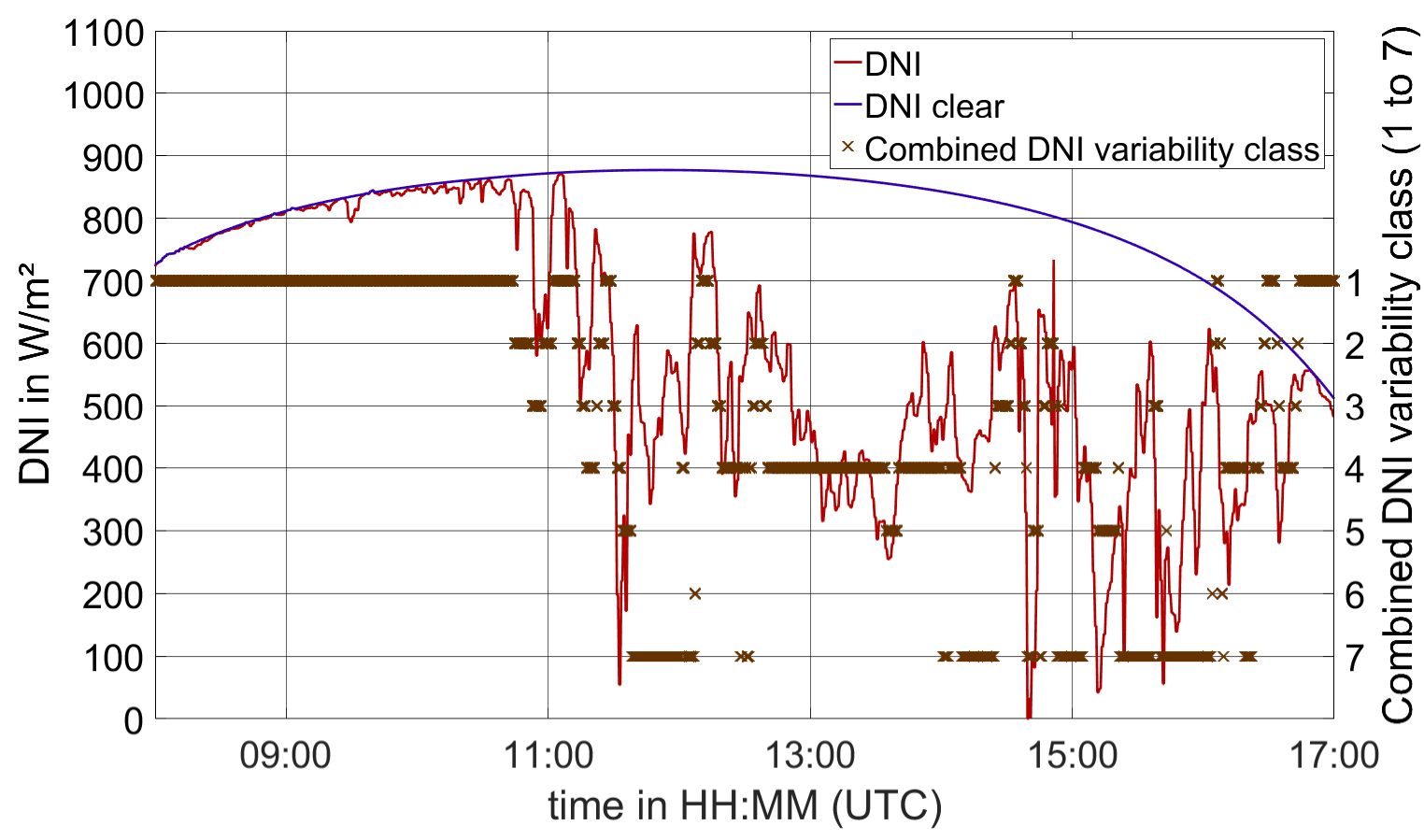

Figure 11: Combined DNI variability classes of an example day (09.09.2015)

421 In section 5, two new solar field control strategies with optimized specific control parameters for 422 each of the 7 combined DNI variability classes will be presented.

4235 Introducing and benchmarking DNI variability class dependent $424 \quad$ solar field controllers

\subsection{DNI variability class dependent solar field controllers}

426 The solar field control system implemented in VSF is composed of local collector temperature 427 controllers for each of the SCAs and a field main flow controller. A detailed description of the 428 control system is published in Noureldin et al. 2019a and Noureldin et al. 2019b. In this work 429 we present only a brief summary. 
430 The local collector temperature controller regulates the HTF temperature in the receiver tubes

431 and ensures that it does not exceed the tolerated temperature limits as defined by the

432 manufactures. This is achieved by manipulating the collector angle using a proportional-integral

$433(\mathrm{PI})$ temperature feedback $(\mathrm{FB})$ controller to ensure that the SCAs are tracking the sun and

434 defocusing in case the temperature set point of the SCA is exceeded. Each SCA gets an

435 individual temperature set point which is derived from the actual field inlet temperature and load.

436 In addition, there is an emergency service function, which defocuses completely the

437 corresponding SCA if the temperature limits of the HTF are exceeded.

438 Solar field outlet temperatures can be regulated by the solar field inlet mass flow. This task is 439 handled by the main solar field flow controller. The mass flow can be adjusted by the applied 440 differential pressure trough the HTF feed pump. The implemented control concept includes a 441 feed forward (FF) pump controller, a temperature feedback (FB) controller and a focus feedback 442 loop. For the FF part, the required mass flow and, thus, pump pressure is calculated by means 443 of an energy balance over the field fed by the current overall DNI. The DNI value is the average 444 of two pyrheliometer measurements as used in the real power plant (reference controller). Any 445 systematic offsets induced by the FF part are corrected by a temperature FB controller. This 446 controller uses as input the field outlet temperature set-point and operates with an adaptable 447 time constant adjusted to the throughput time of the solar field. Especially in volatile irradiance 448 situations, the large time constant of the solar field makes it challenging to avoid temperature 449 overshoots in associated loops which often trigger defocusing of one or more SCAs in the loops. 450 The focus feedback controller forces the collectors to refocus by increasing the mass flow if 451 overall defocusing states of the field increases. 
452 The general form for a PI-controller for variable $u(t)$ in response to an error $e(t)$ in the process 453 variable from the set-point is

$$
u(t)=K_{c} e(t)+\frac{K_{c}}{T_{i}} \int e(t) d t
$$

Equation 8

454 The behavior of the local collector focus and the field main flow PI controllers can be adjusted 455 according to the first order plus dead-time method. This is achieved by deriving the controller 456 gain, $K_{c}$, and integral time, $T_{i}$, in Equation 8 from the process gain $K_{p}$, time constant $T_{p}$, process 457 dead time $\theta_{p}$, such that

$K_{c}=\frac{1}{K_{p}} \frac{T_{p}}{\left(\theta_{p}+\omega T_{p}\right)}$. Equation 9

458 The controller gain could be further manipulated by introducing the factor $\omega$ varying from 0.1 to 45910 for aggressive to conservative controller behavior, respectively. The integral time, $T_{i}$, is set to 460 the process time constant, $T_{p}$. The implemented reference VSF controller uses control 461 parameter as defined in Table 4 according to Noureldin et al. 2019b. The reference controller is 462 designed to emulate state of the art solar field control while eliminating the need for manual 463 intervention from the solar field operators. The controller has proven to work robustly in various 464 weather conditions. 


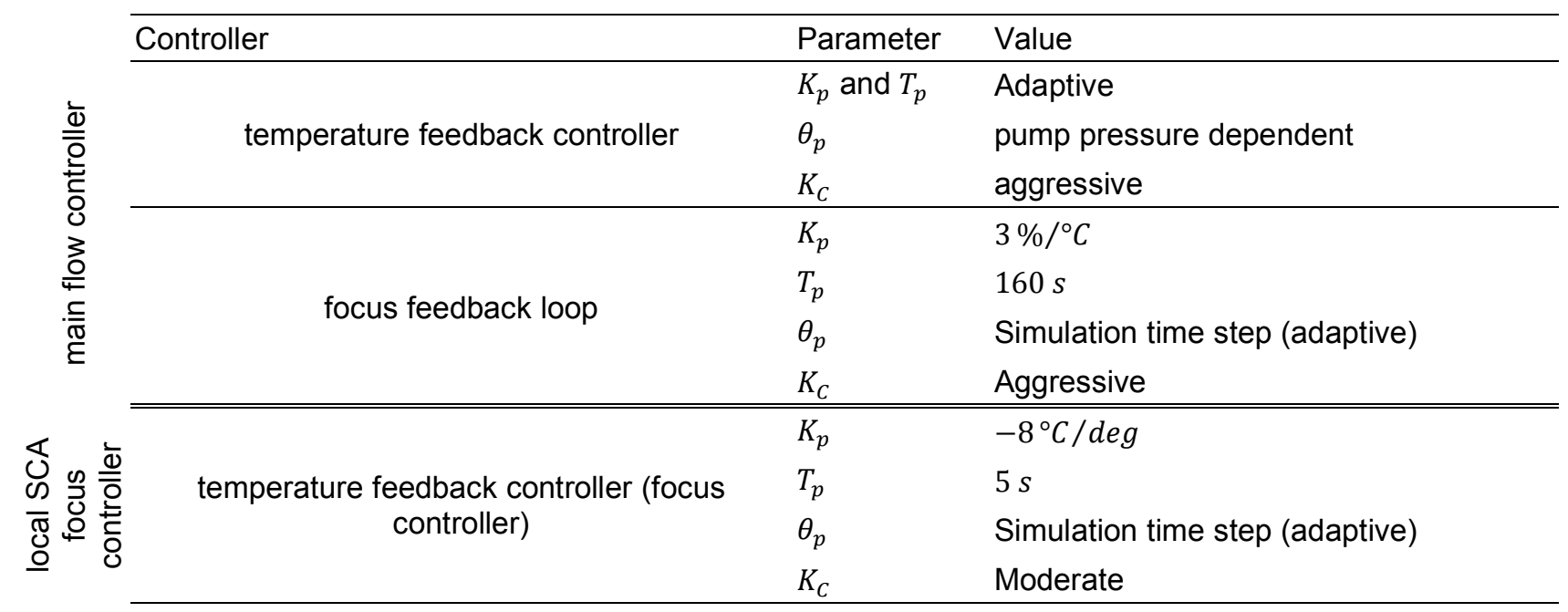

471 The controller parameters presented in Table 4 are utilized as the reference case of a field 472 controller not taking into account information on spatial DNI distribution or DNI variability. In this 473 work, two new control strategies have been developed taking into account the information 474 available from the nowcasting system (spatial DNI field average and DNI variability). The 475 concept is to use different values for the controller parameters depending on the irradiance 476 situation. A clear sky situation e.g. can be operated with a more aggressive controller than a 477 strongly fluctuating situation. The adaptation of controller parameters is applied to the main flow 478 controller and to the local focus controllers. For the class dependent controller, an optimized set 479 of control parameters is defined for each of the seven combined DNI variability classes (see 480 section 4.3). The trimming of the controller parameters has been carried out for a number of test 481 situations and the parameters which best meet the criterion are selected. The first new controller 482 is trimmed to maintain the solar field outlet temperature as constant as possible whereas the 483 second one tries to maximize the solar heat collection by minimizing any defocusing. The 484 controller tuning is based on manual tuning and on the Ziegler-Nichlos method (Schlichting 485 2018). For the remaining document we will call these controllers OT (objective temperature) and 486 OFR (objective focus rate). The optimized process and control parameters are listed in Table 5 
487 and Table 6. Whereas the $K_{p}$ and $T_{p}$, values of the controller are pre-defined (for the focus

488 controller) or adaptively calculated for the current field situation (for the temperature FB

489 controller), the trimming is realized by modifying the tuning factor $\omega$ resulting in a conservative,

490 moderate or aggressive tuning.

Table 5: Adjusted process and control parameters of the class dependent controller OT temperature FB controller focus FB loop temperature FB controller (focus controller)

\begin{tabular}{|c|c|c|c|c|c|c|c|c|c|}
\hline \multirow[b]{2}{*}{ class } & & & & & & & \\
\hline & $K_{p}$ & $T_{p}$ & $K_{C}$ & $K_{p}$ & $T_{p}$ & $K_{C}$ & $K_{p}$ & $T_{p}$ & $K_{C}$ \\
\hline 1 & adaptive & adaptive & moderate & $3 \% /{ }^{\circ} \mathrm{C}$ & $160 s$ & $\begin{array}{l}\text { Very } \\
\text { aggressive }\end{array}$ & $-2{ }^{\circ} \mathrm{C} / \mathrm{deg}$ & $50 \mathrm{~s}$ & moderate \\
\hline 2 & adaptive & adaptive & aggressive & $3 \% /{ }^{\circ} \mathrm{C}$ & $160 s$ & aggressive & $-2^{\circ} \mathrm{C} / \mathrm{deg}$ & $50 s$ & moderate \\
\hline 3 & adaptive & adaptive & moderate & Deactivated & deactivated & deactivated & $-2{ }^{\circ} \mathrm{C} / \mathrm{deg}$ & $50 \mathrm{~s}$ & aggressive \\
\hline 4 & adaptive & adaptive & moderate & Deactivated & deactivated & deactivated & $-2^{\circ} \mathrm{C} / \mathrm{deg}$ & $50 \mathrm{~s}$ & aggressive \\
\hline 5 & adaptive & adaptive & aggressive & Deactivated & deactivated & deactivated & $-2{ }^{\circ} \mathrm{C} / \mathrm{deg}$ & $50 \mathrm{~s}$ & moderate \\
\hline 6 & adaptive & adaptive & aggressive & Deactivated & deactivated & deactivated & $-2{ }^{\circ} \mathrm{C} / \mathrm{deg}$ & $50 s$ & aggressive \\
\hline 7 & adaptive & adaptive & aggressive & Deactivated & deactivated & deactivated & $-2^{\circ} \mathrm{C} / \mathrm{deg}$ & $50 \mathrm{~s}$ & aggressive \\
\hline
\end{tabular}

Table 6: Adjusted process and control parameters of the class dependent controller OFR temperature FB controller focus FB loop temperature FB controller (focus controller)

\begin{tabular}{|c|c|c|c|c|c|c|c|c|c|}
\hline class & $K_{p}$ & $T_{p}$ & $K_{C}$ & $K_{p}$ & $T_{p}$ & $K_{C}$ & $K_{p}$ & $T_{p}$ & $K_{C}$ \\
\hline 1 & adaptive & adaptive & moderate & $3 \% /{ }^{\circ} \mathrm{C}$ & $160 s$ & $\begin{array}{l}\text { Very } \\
\text { aggressive }\end{array}$ & $-6^{\circ} \mathrm{C} / \mathrm{deg}$ & $115 s$ & moderate \\
\hline 2 & adaptive & adaptive & aggressive & $3 \% /{ }^{\circ} \mathrm{C}$ & $160 s$ & aggressive & $-6^{\circ} \mathrm{C} / \mathrm{deg}$ & $115 s$ & moderate \\
\hline 3 & adaptive & adaptive & conservative & deactivated & deactivated & deactivated & $-6^{\circ} \mathrm{C} / \mathrm{deg}$ & $115 s$ & moderate \\
\hline 4 & adaptive & adaptive & moderate & $3 \% /{ }^{\circ} \mathrm{C}$ & $160 \mathrm{~s}$ & moderate & $-6^{\circ} \mathrm{C} / \mathrm{deg}$ & $115 s$ & moderate \\
\hline 5 & adaptive & adaptive & conservative & $3 \% /{ }^{\circ} \mathrm{C}$ & $160 \mathrm{~s}$ & moderate & $-6^{\circ} \mathrm{C} / \mathrm{deg}$ & $115 s$ & moderate \\
\hline 6 & adaptive & adaptive & aggressive & deactivated & deactivated & deactivated & $-6^{\circ} \mathrm{C} / \mathrm{deg}$ & $115 s$ & moderate \\
\hline 7 & adaptive & adaptive & aggressive & deactivated & deactivated & deactivated & $-6^{\circ} \mathrm{C} / \mathrm{deg}$ & $115 s$ & moderate \\
\hline
\end{tabular}

\subsection{Selection of assessment days}

494 The benchmark is performed over 22 days distributed over the year 2015 at PSA. The data set

495 is chosen in such a way, that it includes a wide variety of conditions in irradiance, cloud height

496 and type (low layer, middle layer, high layer and multi-layer conditions) as well as the resulting

497 DNI variability classes (see Figure 12). The cloud height information is shown since it is one of

498 the main uncertainty contributors of ASI systems (Nouri et al. 2019b; Nouri et al. 2019c). Cloud

499 height measurements are taken by a CHM 15 k Nimbus ceilometer from G. Lufft Mess- und

500 Regeltechnik GmbH. 


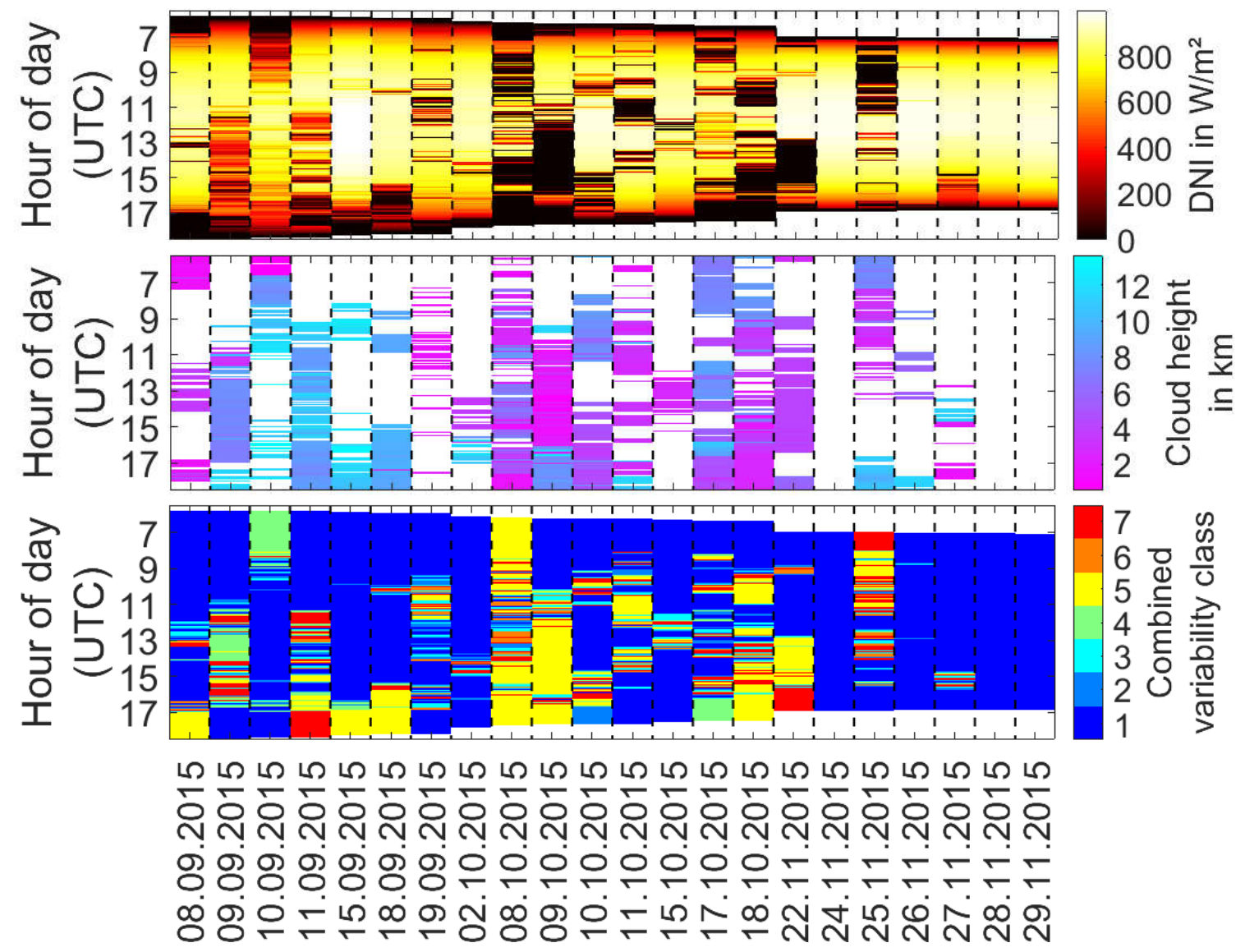

Figure 12: Pyrheliometer based DNI, cloud height as measured by a ceilometer and combined DNI variability class over

the 22 test days

504 Comparing the distribution in combinations of spatial and temporal DNI variability class during 505 the 22 test days (see Figure 13), it reveals that the 22 days include almost all combinations of 506 the 2 year period as analyzed in section 4.3. Only 6 combinations which exist in the two year 507 period are missing in the 22 days test period. These 6 combinations describe rather rare 508 transient conditions which make less than $0.37 \%$ of the entire 2 year data set. Overall the 22 509 days include with $19.2 \%$ a higher share of the intermediate to highly variable conditions (spatial 510 class 3 or 4 and temporal class 3 to 7 ), compared to $13 \%$ in the 2 year data set. In turn, less 511 interesting overcast conditions (spatial class 5 and temporal class 6 to 8 ) have only a share of $5127.8 \%$ compared to $14.8 \%$ within the 2 years. For the remaining combinations the 22 days 513 resemble the two year data set very well. 


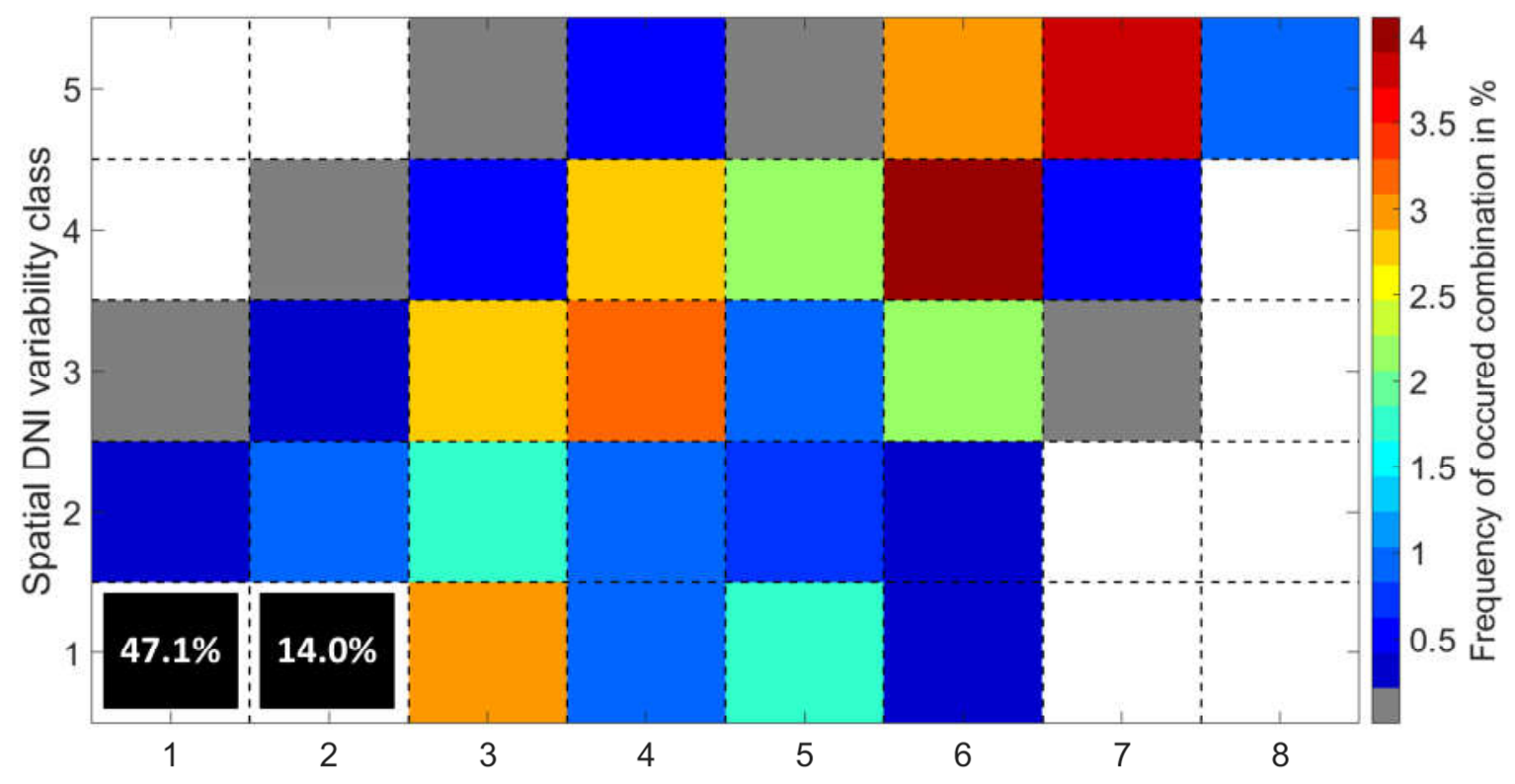

Temporal DNI variability class

Figure 13: Temporal and spatial DNI variability class distribution of possible combinations within 22 test days.

Combinations spatial class $1 \&$ temporal classes 1 or 2 account for more than $61 \%$ of the data set. These bins are not described by the colorbar for a better readability of the remaining combinations.

519 In this section the two new class dependent solar field controllers are benchmarked against the 520 state of the art reference controller based on the La Africana power plant design. Five different 521 criteria are used to evaluate the solar field controller's performance.

522 - Overall expected revenues in $€$ between the new class dependent controller and the 523 reference controller.

524 - The accumulated solar field thermal heat flow

525 - Average solar field focus rate as relative value. It accounts to $100 \%$, if all collectors of the $526 \quad$ solar field are fully focused.

- RMSD of the solar field outlet temperature with the design set temperature as reference. 
- Reduction of emergency defocus incidents: Relative value which compares whether or not the new class dependent controller is capable of reducing emergency defocus incidents with respect to the reference controller. Emergency defocus incidents occur when the fluid temperature exceeds a maximum safety threshold indicated by the fluid

\section{3} manufacturer.

534 performance. The other parameters contribute to a better understanding of the controller's

535 individual behavior. The spider plot in Figure 14 illustrates the overall results for these five

536 criteria. Only relative changes to the reference controller are depicted, where positive values

537 indicate an improvement and negative values a decline in performance in a particular criteria.

538 For example, the calculation of change in relative revenue is always calculated according to 539 Equation 10

$c_{\text {rel.rev }}=\left(\right.$ rev $_{\text {classDep }} /$ rev $\left._{\text {ref }}-1\right) \cdot 100 \%$ Equation 10

540 ,where rev $_{\text {ref }}$ is the revenue of the reference controller and $r e v_{\text {classDep }}$ is the corresponding 541 revenue of the class dependent controller.

542 The class dependent controller OT outperforms the reference controller in all criteria. In terms of 543 overall revenue, the controller OT outperforms the reference controller by $1.38 \%$.

544 A particularly significant improvement can be seen by the controller OT for the criteria reduction 545 of emergency defocus incidents with $18.6 \%$. Emergency defocus incidents can lead to strong 546 temperature transients within collectors (temperature gradients above $40^{\circ} \mathrm{C}$ in 3 minutes are 547 possible), which affect the overall system stability, solar field outlet temperature and focus rate.

548 This reduction of emergency defocus incidents by itself is already an important improvement. 
549 Emergency defocus incidents are triggered by exceeding maximum safety threshold

550 temperatures within the collectors. The emergency defocus means first of all that additional

551 operations of the collector drives and rotation and expansion performing assemblies (REPA) are

552 necessary, which could accelerate degrading effects. Furthermore, frequent temperature

553 transients, especially with temperatures by several degrees above the set point, could affect the

554 lifetime of REPAs, HTFs, receiver tubes and all other components exposed to the transients.

555 Since sufficient experience on the degradation mechanisms is not yet available, the findings

556 cannot be directly translated into economic figures.

557 The class dependent controller OFR reaches a revenue increase of $1.40 \%$, nearly identical to 558 the increase for OT. However, while OFR also outperforms the reference in focus rate and heat 559 flow, it is outperformed by the reference in terms of RMSD temperature (by $-5.4 \%$ ) and 560 emergency defocus incidents (by $-0.6 \%$ ). Despite the objective of this controller it performs only 561 marginally better than the controller OT in focus rate with an improvement of $1.62 \%$ compared to 562 an improvement of $1.48 \%$. This can be partly explained by the significantly higher number of 563 emergency defocus incidents compared to the controller OT. Each emergency defocus incident 564 leads to a short-term total defocusing of a collector and in turn to additional temperature 565 transients which is reflected by the increased RMSD. This leads to a stronger penalization of the 566 controller OFR compared to the controller OT and thus to a similar improvement in revenue, 567 despite the higher overall heat flow for OFR. Increased number of incidents is caused by the 568 target of the controller. The higher number of emergency defocus incidents is caused by the 569 controller OFR objective. In order to keep the focus rate as high as possible, the controller OFR 570 uses predominantly the pump pressure as control variable, intending to adapt the mass flow to 571 the prevailing conditions while maintaining a constant high focus rate. This approach usually 572 leads to a higher heat flow, yet, it is more vulnerable under variable quickly changing conditions 
573 in which adaptation of the focus rate are inevitable. A partially too late intervention of the local

574 SCA focus controller gives less leeway for countermeasures, which in turn leads to a higher rate 575 of emergency defocus incidents.

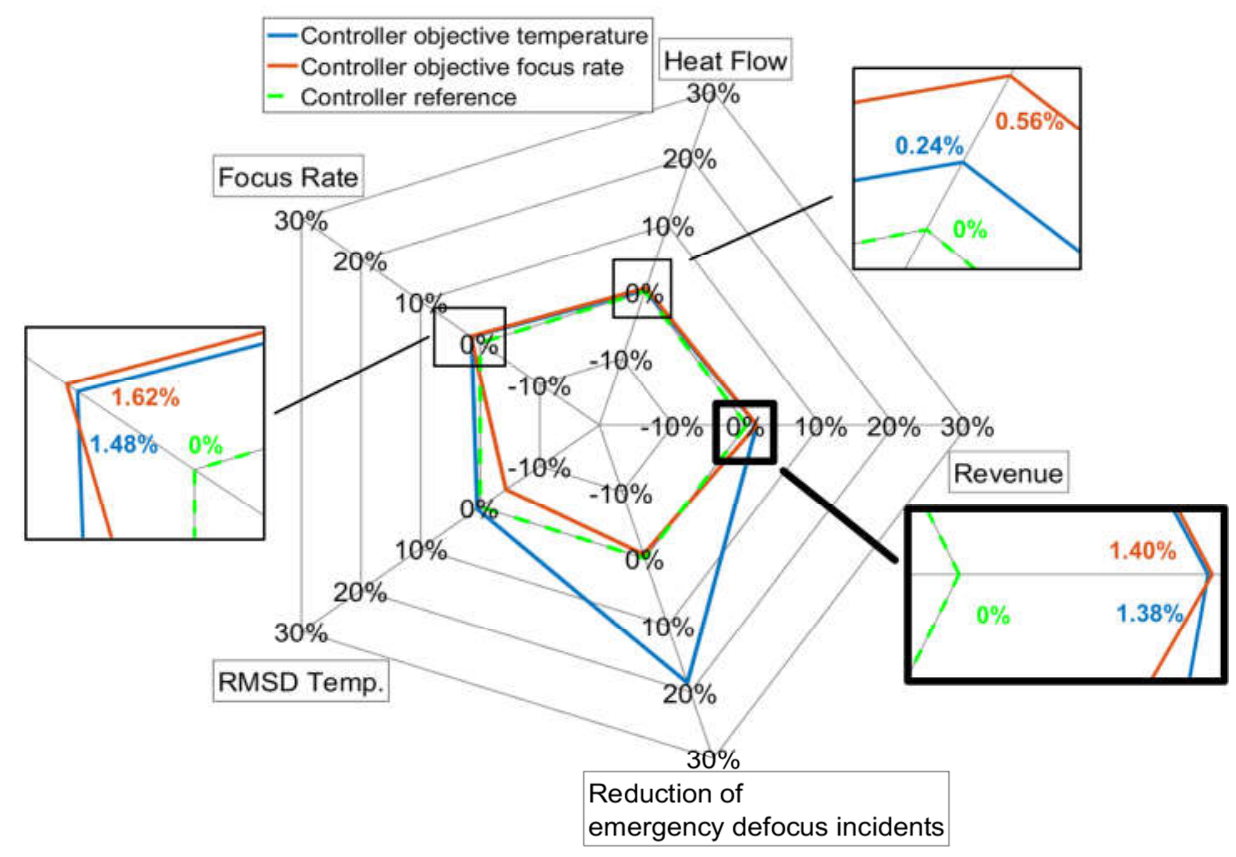

Figure 14: Comparison of the three controller setups reference, objective temperature, objective focus rate over 5 criteria and based on 22 test days. Relative changes compared to the reference controller are plotted. Positive values indicate an improvement and negative values a decline in performance in a particular criterion.

580 For each day, the absolute revenue and the change of relative revenue between the reference

581 controller and the two class dependent controller are compared in Figure 15. Both class

582 dependent controllers outperform the reference controller in 20 out of 22 days. The

583 underperforming days are characterized by longer time periods with fast changing variability

584 classes (see Figure 12). How such an underperformance can be avoided is discussed in the 585 next section.

586 It is observed that the potential gains in revenue are rather different between all days. The 587 lowest revenue is expected on the 25.11.2015 with roughly $7.9 \mathrm{k} €$ (reference control) and the 
588 highest on the 15.09.2015 with roughly $98.8 \mathrm{k} €$ (reference control). Over all 22 days the absolute 589 benefit of the new class dependent controller amounts to roughly $15 \mathrm{k} €$ for the controller OT and 590 roughly $15.2 \mathrm{k} €$ for the controller OFR with an overall absolute revenue of roughly $1,083.3 \mathrm{k} €$ 591 (reference controller). This is an increase of about $1.4 \%$ in revenue for the new class dependent 592 controller concept.

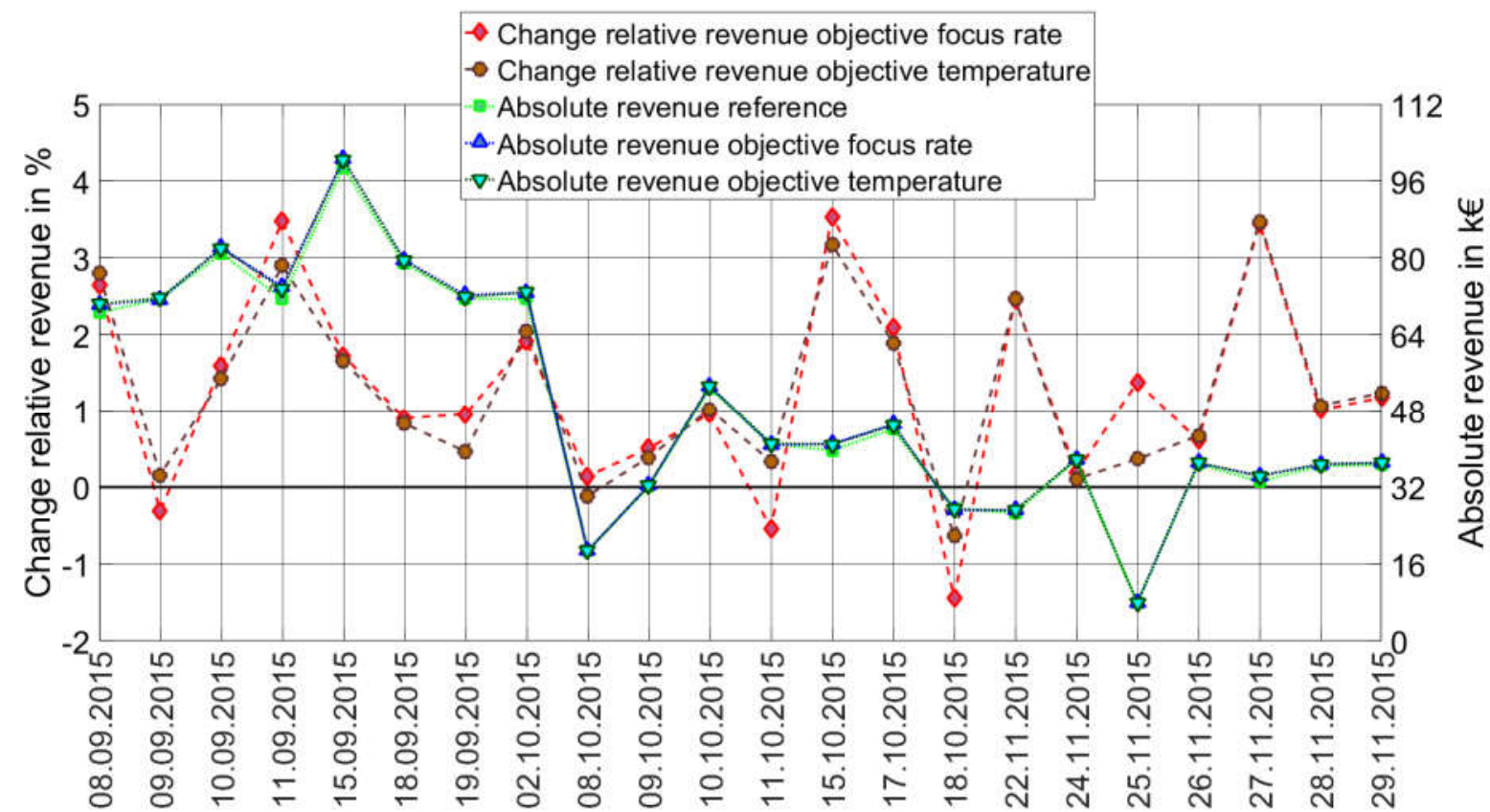

Figure 15: Absolute and relative daily revenue for reference controller and the two class dependent controllers.

\section{Detailed performance assessment and applicability conditions}

\subsection{Identification of applicability conditions}

597 In section 5.3 we investigated the benefit of the class dependent controller compared to the

598 reference controller. These analyses looked at the overall benefit over the entire data set or over

599 complete days. A clear benefit is visible in terms of revenue. Yet, it might be the case that the

600 class dependent controller will be outperformed by the reference controller under certain

601 conditions. 
602 In this section, we discretize the entire data set into short data packages and analyze the impact 603 of the combined DNI variability classes as well as the combinations of spatial and temporal DNI 604 variability classes on the expected revenue. The goal is to identify a binary decision system, 605 which will decide if either a class dependent controller or the reference controller is preferable 606 for the prevailing conditions. We call this binary decision system as the identification of 607 applicability of the ASI derived DNI variability class and the enhanced controller.

608 A DNI variability classification update is performed every $30 \mathrm{~s}$, according to the ASI system 609 resolution. The spatial DNI variability classification uses information of the past 5 minutes and 610 the temporal classification of the past 15 minutes. For the identification of applicability 611 conditions, we discretize the 22 days in sliding data packages of 10 minutes duration. The 612 resolution of these sliding data packages corresponds to the temporal resolution of the ASI 613 system of 30 seconds. Each data package time stamp corresponds to the end of the package. 614 The 22 days result in roughly 20000 data packages. These data packages enable a high 615 temporal resolution impact analysis of the actual DNI variability classes on the controllers. No 616 aggregation over the data packages is done. An aggregation would lead to an artificial repetition 617 of the same time stamps within the summed up values.

618 Considering the solar field time constant of approximately $10 \mathrm{~min}$, it is obvious that 10 minute 619 data packages might be too short to fully cover some of the processes which unfold more slowly 620 within some parts of the extensive solar field. This leads to the situation that a certain analysis 621 window is not only affected by the DNI situation during this time window but also from the DNI 622 situation and the controller activity in the previous time periods. Especially when DNI classes 623 rapidly change along the analysis windows, exist no one to one mapping of classification to 624 controller result. Nevertheless, the used 10 minute time windows cover many occurring 
625 processes. Longer time windows would corrupt the intention of this analysis, since they would 626 again average out effects we want to work out.

627 In section 6.2 we will present the benefit of the applicability conditions according to the results 628 presented in section 5.3 without the discretization in sliding data packages.

629 The change in relative revenue of all data packages is discretized and averaged within the 630 combined DNI variability classes (see Figure 16). The combined class 1 describes the dominant 631 sunny conditions within the data set with an occurrence of $67 \%$. The controller OT shows an 632 advantage compared to the reference controller for the classes 1, 2, 6 and 7 which account for 633 more than $82 \%$ of the data set. The controller shows a significant disadvantage during combined 634 class 4 conditions, which describe an intermediate spatial variability with a low to intermediate 635 temporal variability. Under these conditions, more than $50 \%$ of the solar field is shaded.

636 The controller OFR shows an even stronger advantage compared to the reference controller for 637 the classes 1, 2, 6 and 7 as well as class 3 which account together for more than $88 \%$ of the 638 data set. However, the class 4 and 5 show a very pronounced disadvantage in revenues 639 compared to the reference. The absolute energetic loss for these classes is comparably low, 640 since these classes are rare and as they have a high shaded solar field share and a low cloud 641 transmittance (especially for class 5). 


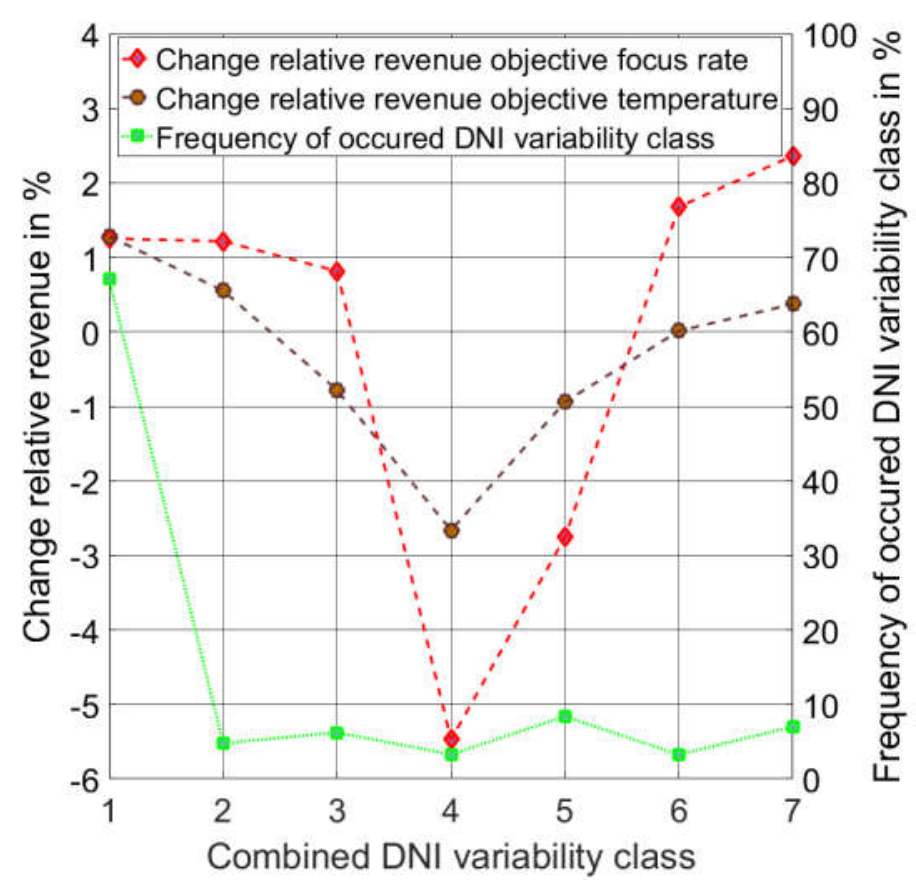

Figure 16: Change relative revenue discretized over combined variability classes and occurrence of classes within the

645 Most of the combined DNI variability classes (with exception of combined class 6) include more 646 than one combination of spatial and temporal DNI variability classes. Therefore, we discretized 647 the change in relative revenue of all data packages in the combinations of spatial and temporal 648 DNI variability class. The results of this analysis are shown in Figure 17, which considers the 649 spatial class on the $y$ axis, the temporal class on the $x$ axis, the change in relative revenue by 650 the color coding and the occurrence of a certain conditions as the stated value within the bins.

651 The class dependent controllers outperform the reference controller in 20 (OT) and 22 (OFR) 652 combinations of spatial and temporal DNI variability conditions (green and cyan bins). They are 653 outperformed in 11 (OT) and 9 (OFR) combinations (red and purple bins), of which 6 are 654 identical for both controllers. The mentioned 20 and 22 combinations of spatial and temporal 655 DNI variability classes account for more than 85\% (OT) and 89\% (OFR) of the data set. Almost 656 all conditions with an advantage for the reference controller are found for highly variable 
657 conditions, which are connected to a low average DNI and plant yield. For each class dependent 658 controller only one underperforming combination of spatial and temporal DNI variability class is 659 found, which can be connected to a high average DNI and yield. However, these combinations 660 account for less than $0.3 \%$ of the data set.

661 The combination of spatial class 5 and temporal class 3 shows for both controllers a change in 662 relative revenue above $20 \%$. On the contrary, the combination of spatial class 3 and temporal 663 class 7 shows for both controllers a change in relative revenue around $-20 \%$. However, both 664 these combination account for less than $0.1 \%$ of the data set and are therefore mostly irrelevant. 665 For the remaining combinations the change in relative revenue is clearly within $\pm 10 \%$ (mostly $666 \pm 5 \%)$.

667 The results depicted in Figure 17 are utilized for the identification of the applicability of the class668 dependent controller. For each combination of spatial and temporal DNI variability class with an 669 expected benefit in revenue, it makes sense to use the class dependent controller whereas the 670 reference controller is recommended for the remaining combinations. Thus, this matrix defines

671 the applicability of the class dependent controller giving the name to the overall concept of 672 applicability classes for the solar field control. 


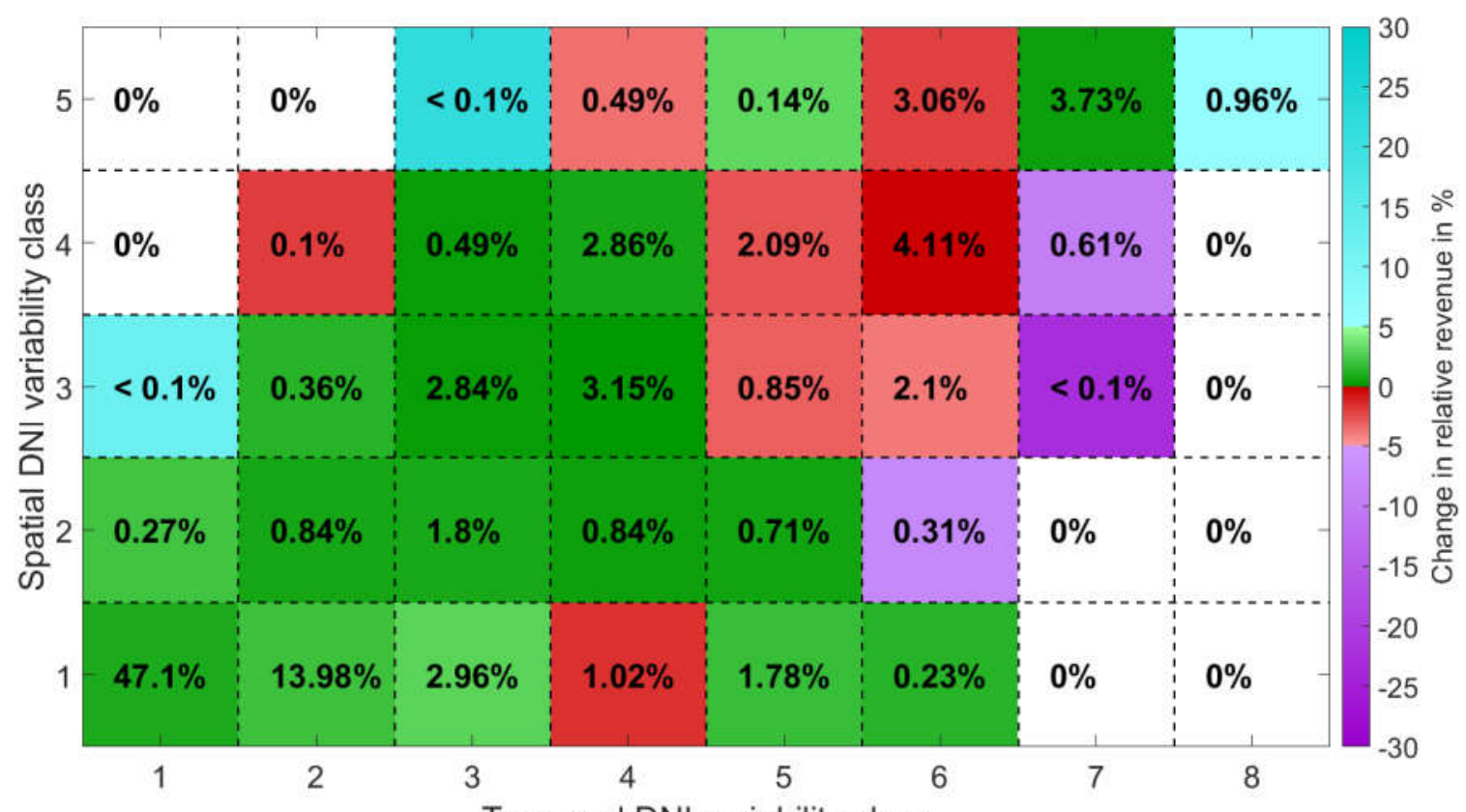

674

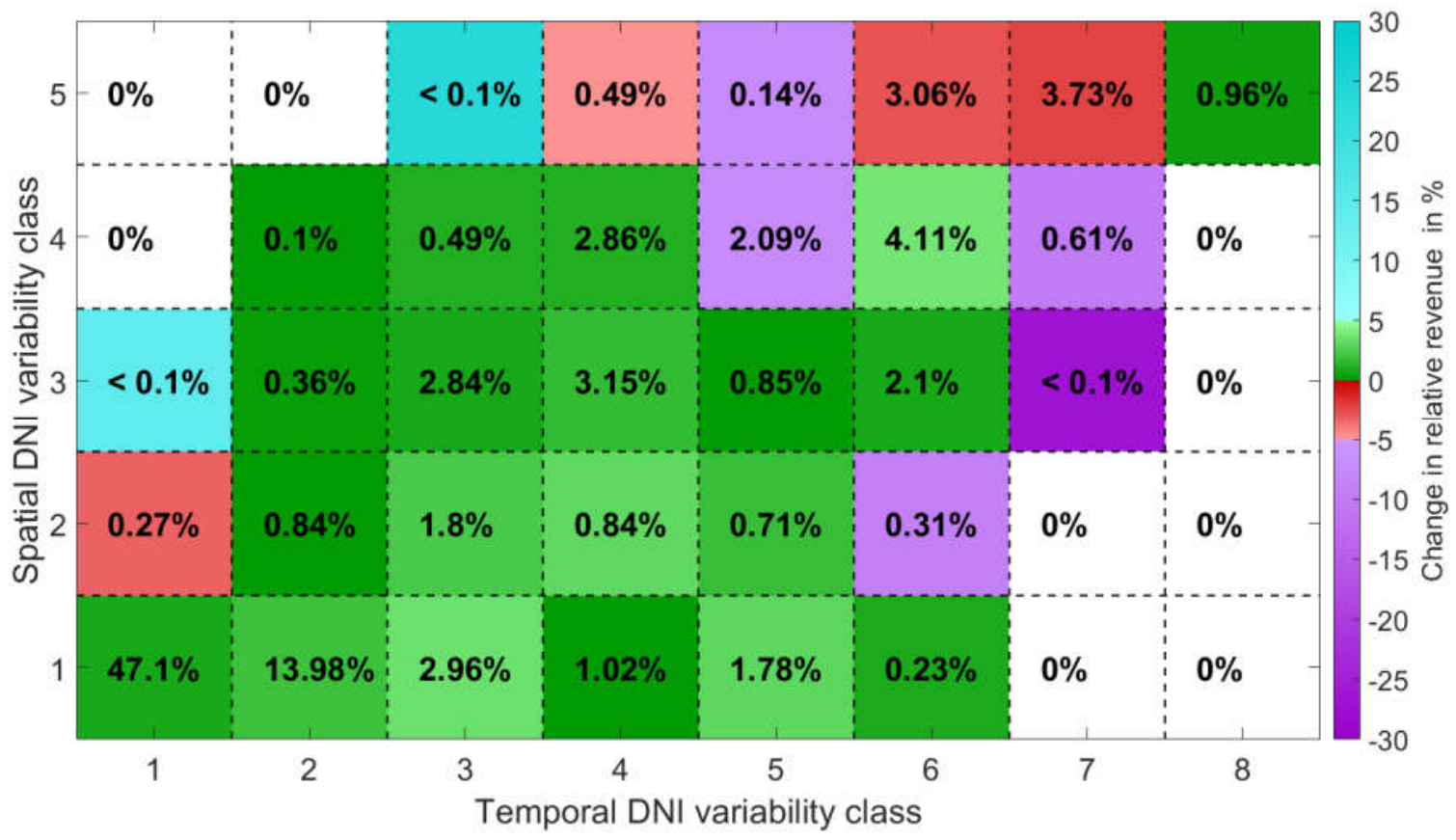

676 Figure 17: Change in relative revenue discretized in combinations of temporal and spatial DNI variability classes. The color coding describes the relative change in revenue within each bin. The occurrence of each bin is indicated by the stated values. (top) controller OT (bottom) controller OFR

680 In this section we describe how the above findings can be used to create an improved

681 hybridized control strategy. The hybridized control concept consists of two sets of controllers. 
682 The first one is the reference controller making use of the irradiance information from the two 683 pyrheliometers whereas the second one uses the class dependent control parameters and 684 additional information from the ASI system. The ASI information delivers the DNI average over 685 the field as well as the classification into temporal and spatial DNI classes. The applicability 686 matrix defined in the last section is used to decide whether the reference controller or the class 687 dependent controller is used in the current DNI situation represented by the class derived from 688 the ASI information.

689 All 22 days are evaluated once again with the VSF using this hybridized control strategy for both 690 class dependent controllers. We will continue to call the hybridized controller class dependent 691 controller with the objective temperature (OT) and focus rate (OFR), since for the hybrid 692 operation the class dependent controller account for the predominant share of the data set. 693 Compared to the analysis presented in section 6.1, the applied controller is now continuously 694 selected based on the class whereas in the former study the same controller was used 695 throughout the day only adapting its parameters according to the prevailing class. In a first step, 696 we analyze the performance based on the 10 min sliding data packages according to section 697 6.1. The improvement due to the class dependent controllers with regards to the applicability 698 conditions is evident, when looking into the overall change in relative revenue discretized over 699 the combined temporal and spatial DNI variability classes (see Figure 18). The controller OT 700 shows a significant increase especially for the classes 2, 3, 4, 6 and 7 . This holds especially for 701 class 4 where the relative revenue changes from a decrease of $-2.7 \%$ to an increase of $4.6 \%$. 702 Nonetheless, we see also a slight decrease for class 5 . The controller OFR shows a significant 703 increase within all classes. For class 4 and 5 , the previous reductions of $-5.5 \%$ and $-2.8 \%$ in 704 revenues increase to $5.1 \%$ and $3.5 \%$, respectively. 


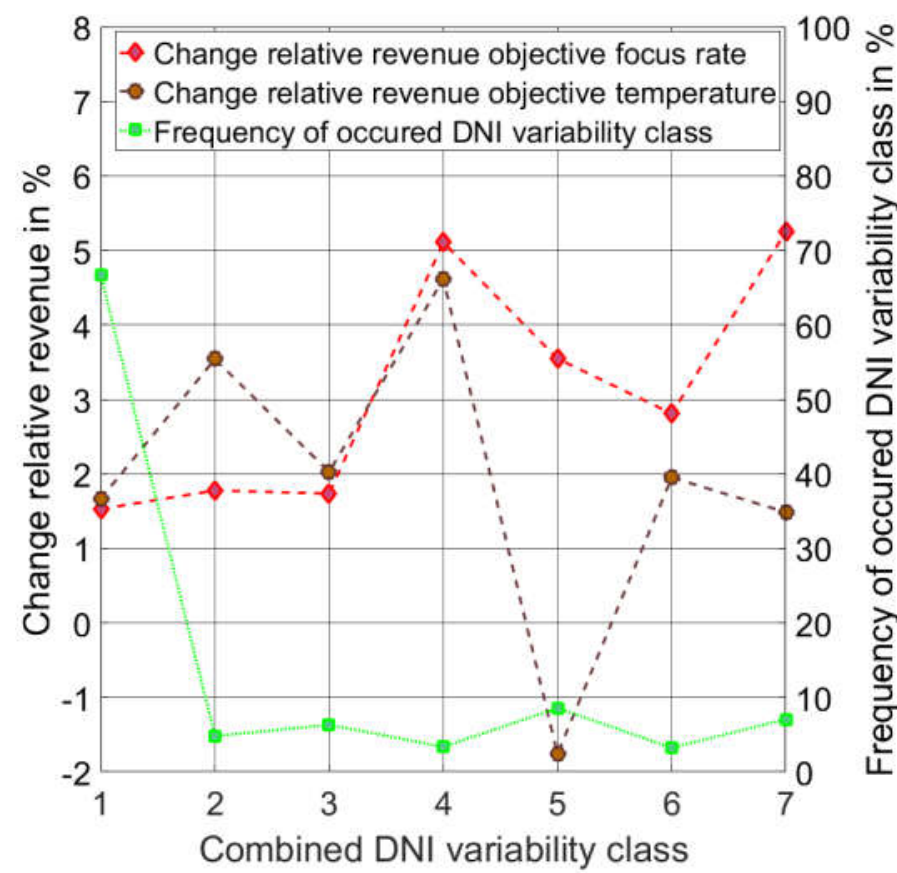

Figure 18: Change relative revenue discretized over combined variability classes and occurrence of classes within the

708 Looking into the change in relative revenue discretized over the combinations of spatial and

709 temporal DNI variability classes, we observe that both class dependent controllers outperform

710 the reference controller in 25 combinations. They are only outperformed in 6 combinations,

711 which account for $9 \%$ (OT) and 3.2\% (OFR) of the data set (see Figure 19). Four of these six

712 unfavorable combinations are identical for both controllers. The considerable higher share in the

713 case of controller OT is due to the two combinations spatial 5 and temporal 6 and 7 . Each of

714 these combinations has a share above $3 \%$ of the data set. Overall, the absolute energetic effect

715 of these combinations is insignificant as they refer to almost fully shaded low DNI conditions.

716 These two combinations also explain the slightly negative impact in combined DNI variability

717 class 5 on the controller OT (see Figure 18). 

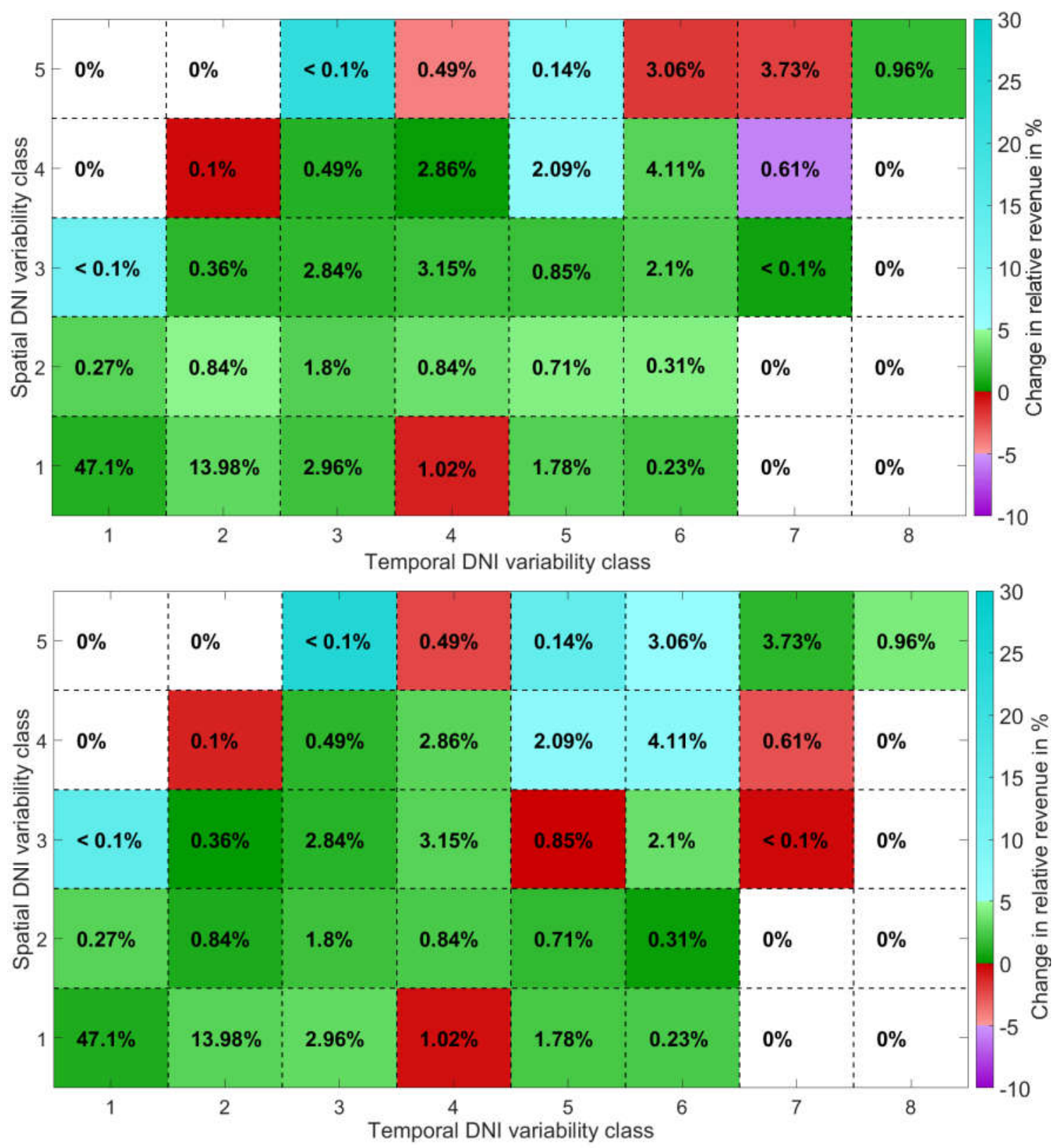

720 Figure 19: Change in relative revenue discretized in combinations of temporal and spatial DNI variability classes for

721 hybridized controllers using the applicability. The color coding describes the relative change in revenue within each 722 bin. The occurrence of each bin is indicated by the stated values. (top) hybridized controller OT using the identified applicability (bottom) hybridized controller OFR using the identified applicability

724 In a next step we analyze the impact of the applicability conditions on the overall results 725 according to section 5.3, without the discretization of sliding data packages in DNI variability classes. Figure 20 shows the change in relative and absolute revenues discretized over the 22 
727 days. Both class dependent controllers show a significant improvement compared to the results 728 presented in section 5.3. The overall absolute revenue compared to the reference controller 729 rises by roughly $20.9 \mathrm{k} €(\mathrm{OT})$ and roughly $21.2 \mathrm{k} €$ (OFR) with the hybridized concept. Without 730 the continuous selection between reference and class dependent controller these improvements 731 were considerably lower with roughly $15 \mathrm{k} €(\mathrm{OT})$ and $15.2 \mathrm{k} €(\mathrm{OFR})$. For the hybrid concept, the 732 reference controller outperforms the controller OT only on the 08.10 .2015 and the controller 733 OFR on the 25.11.2015. These days with roughly $18.7 \mathrm{k} €(08.10 .2015$ reference controller $)$ and $7347.9 \mathrm{k} €(25.11 .2015$ reference controller) have the lowest overall absolute daily revenue. On 73508.10 .2015 , the combined variability class 5 accounts for $57 \%$ of the day (see Figure 12). As we 736 see in Figure 18, the reference controller outperforms the class dependent controller OT by 737 roughly $1.8 \%$ within the combined DNI variability class 5 . Therefore, it is not surprising that the 738 reference controller outperforms the controller OT on this day. The 25.11.2015 is a highly 739 complex day with multi-layer cloud conditions (see Figure 12). This day is responsible for $27 \%$ of 740 all emergency defocus incidents (reference controller) during the 22 days. As we discussed in 741 section 5.3, the controller OFR is much more vulnerable for emergency defocus incidents 742 compared to the controller OT. This explains the strong deviation in revenue change from $+8.8 \%$ 743 (OT) to $-2.1 \%$ (OFR). Concerning the high fluctuations of the relative values, it should not be 744 forgotten that this day accounts to an absolute revenue of only $7.9 \mathrm{k} €$ (reference controller). 


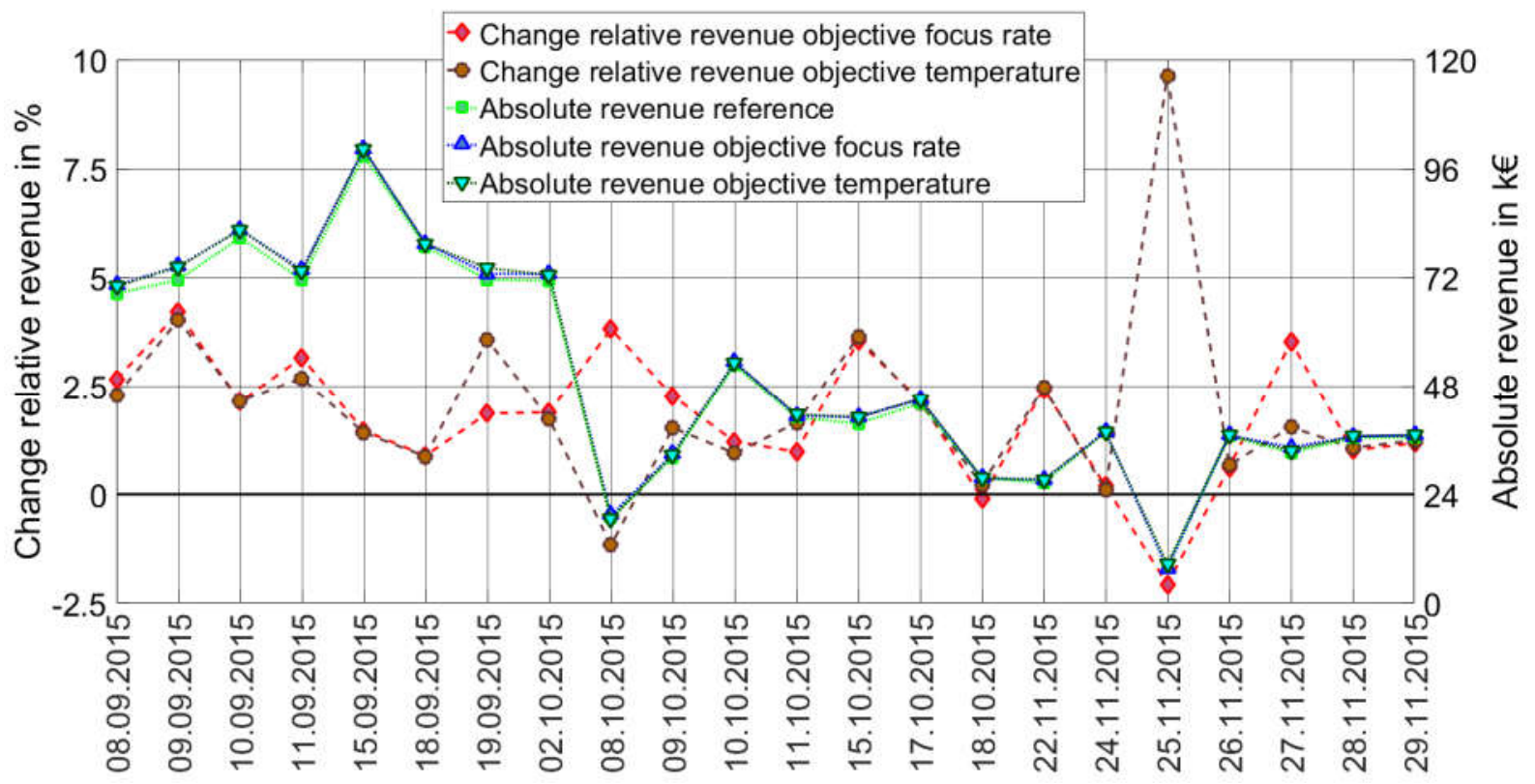

Figure 20: Absolute and relative daily revenue for the reference controller and the two hybridized class dependent

749 Figure 21 illustrates the overall influence of the controllers with regards to the applicability

750 conditions over the previously introduced five criteria. The class dependent controllers

751 outperform the reference controller in all criteria. The advantages and disadvantages of both

752 class dependent controllers are almost completely balanced in terms of revenue, with a benefit

753 above $1.9 \%$ for both class dependent controllers compared to the reference controller.

754 Especially the controller OT shows a significant improvement in the criteria RMSD temperature

755 and reduction of emergency incidents by $9.5 \%$ and $21 \%$, respectively. The controller OFR

756 outperforms the controller OT in terms of heat flow, focus rate and revenue. 


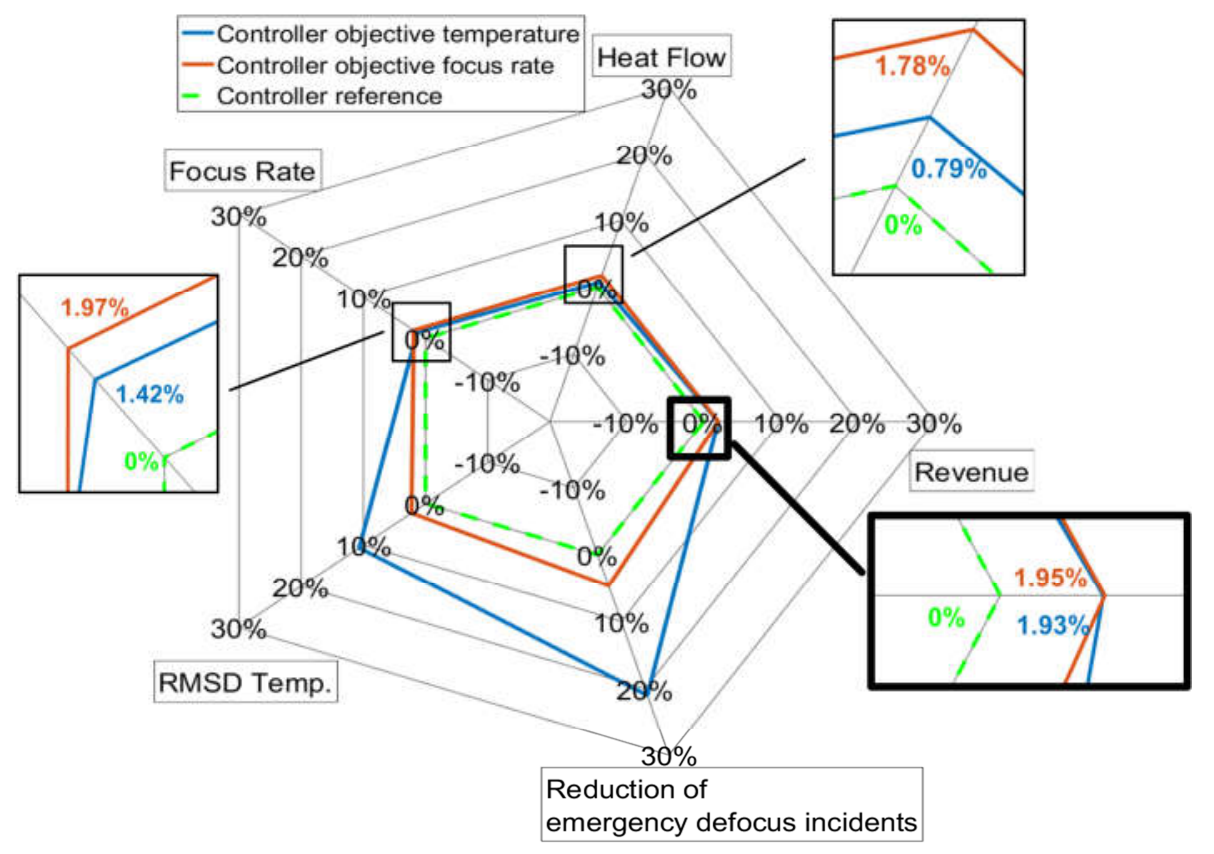

Figure 21: Comparison of hybridized class dependent controllers using applicability against the reference controller 5 criteria and 22 days. Relative changes compared to the reference controller are plotted. Positive values indicate an improvement and negative values a decline in performance in a particular criterion.

\section{Conclusion}

762 We developed a combined temporal and spatial DNI variability classification consisting of 7 763 distinct classes that allows an improved control of parabolic trough power plants. This combined 764 classification procedure includes a temporal DNI variability classification with eight distinct 765 classes as well as a spatial DNI variability classification with 5 distinct classes. Spatial DNI 766 information, provided by an ASI system, are used for the spatial DNI variability classification.

767 Three set ups of controller parameters have been defined based on the same underlying control 768 concept. The reference controller is used as the basis for two modified controller setups. The 769 reference controller parameters for these two additional controllers are trimmed in a way to 770 result in more aggressive or conservative behavior of the controller. Individual trimming values 771 are used for each of the combined DNI variability classes. The two trimmed controller 
772 configurations differ in their behavior. The first has a trend to strongly improve stability the field

773 outlet temperature (called objective temperature, OT) whereas the second tends to increase the

774 overall focusing rate of the field (called objective focus rate, OFR). These new controllers are

775 tailored for a parabolic trough solar field design according to the La Africana 50MW power plant.

776 A benchmark over selected 22 days with variable DNI and different cloud conditions has been

777 performed representing a wide variety of irradiance conditions. The chosen 22 days match the

778 distribution of DNI variability conditions found at PSA over two complete years helping to

779 generate representative results for real situations.

780 The reference controller considers a state of the art solar field controller with DNI information

781 from two pyrheliometers as typically available ground observations in commercial power plants

782 as e.g. the La Africana power plant in Spain. The DNI information is used in a feedforward loop

783 to calculate the expected mass flow required for the actual irradiance. The two other controllers

784 do not use the two meteo station signals but refer to the average DNI values calculated from the

785 DNI map delivered by the ASI system.

786 In the performance assessment study, the actual ruling DNI conditions acting on the solar field 787 are provided by a separate nowcasting system based on shadow cameras, which are looking 788 from above on the same area. These shadow cameras are providing an independent and more 789 accurate estimate of the spatial distribution of DNI. Thanks to the solar towers available at the $790 \mathrm{PSA}$, this unique assessment opportunity is available, while in commercial parabolic trough 791 power plants most likely only the ASI based spatial DNI information will be available.

792 Five different economical and technical performance criteria are compared: the total solar field 793 thermal heat flow, solar field focus rate, RMSD of the solar field outlet temperature (design set 794 temperature as reference), reduction of emergency defocus incidents, and revenue. In terms of 
795 revenue, the major economic criterion, both class dependent controllers are found to be quite 796 similar, despite the higher heat flow of the controller OFR. OFR outperforms the reference by $7971.40 \%$ and OT by $1.38 \%$ if aggregated over the entire 22 day data set.

798 The controller OT outperforms the reference also in the remaining 4 technical performance 799 criteria. In comparison, the controller OFR outperforms the reference controller in focus rate and 800 heat flow, but is outperformed in RMSD of the solar field outlet temperature and reduction of 801 emergency defocus incidents by the reference controller. This explains the close match in 802 revenue between OFR and OT. The higher RMSD of the solar field outlet temperature translates 803 in a higher volatility of the outlet temperature as well as the associated higher number of 804 emergency defocus incidents, which is considered by the revenue calculation trough the 805 penalties.

806 In a subsequent more detailed evaluation step, we evaluated under which conditions the new 807 controllers lead to a revenue increase and whether there exist situations causing a revenue 808 decrease. Increasing revenue conditions are considered as applicable, while the aim is to detect 809 the unfavorable conditions in real time and to avoid using the new controller in such situations.

810 For this evaluation, we discretized the 22 days in 10 minutes sliding data packages.

811 Furthermore, each of these data packets was discretized to one of 40 theoretically possible 812 combinations of spatial and temporal DNI variability classes. The overall relative change in 813 revenue within each combination was analyzed. Around $85 \%$ (OT) and 89\% (OFR) of the data 814 set turned out to be applicable for the new controller.

815 Finally, a hybridized control strategy with regards of the applicability conditions was investigated. 816 Whenever applicable conditions are present, the variability class dependent controllers with DNI 
817 maps are utilized. Otherwise, only the DNI information from two pyrheliometers as input to the 818 reference controller is used.

819 The results of the hybridized control strategy are promising. Both hybridized class dependent 820 controller setups outperform the reference controller in all 5 criteria. In terms of revenue, the 821 reference controller is outperformed by $1.93 \%$ (OT hybridized) and $1.95 \%$ (OFR hybridized), 822 over the entire data set.

823 A significant reduction of emergency defocus incidents $(21 \%)$ is visible for the controller OT 824 (hybridized). This could lead to an additional reduction of maintenance costs, as emergency 825 defocus incidents are related to strong fluctuation in temperature as well as temperatures above 826 safety thresholds, affecting various components within the solar field but also balance of plant. 827 However, these effects cannot be quantified until now.

828 The expected additional absolute benefit in revenue increases to roughly $20.9 \mathrm{k} €$ (OT 829 hybridized) and roughly $21.2 \mathrm{k} €$ (OFR hybridized), over the 22 test days compared to the 830 reference controller.

831 Finally, we conclude that there are significant possibilities to improve the solar field controller of 832 parabolic trough power plants with spatial irradiance information from ASI systems, despite the 833 uncertainties. The variability classification procedure is a crucial element of the concept and 834 reveals the critical information in a suitable manner as needed by the controller. The 835 classification procedure is a substantial requirement to realize the revenue increase, since 836 situation dependent control parameters can be used. The uncertainties of the spatial DNI 837 information might be considerably high at any given moment and for any discrete spot of the 838 solar field. Yet, the ASI system appears to be capable to catch the prevailing overall spatial and 839 temporal DNI variability conditions accurately enough for the plant control. Overall, it is the 
840 classification procedure which uncovers the relevant characteristics of DNI in a proper manner.

841 This became apparent in initial preliminary studies, where the solar field controller had access to

842 the spatial DNI field average from the ASI system, but without the DNI variability classification

843 and the corresponding controller adaptations. Only a slight benefit of few tenth percent in

844 revenue compared to the reference controller were observed in these initial preliminary studies.

845 Theoretically, this slight benefit could be improved, as it is possible to obtain information from

846 the DNI maps with a spatial resolution corresponding to the size of individual SCAs, allowing a

847 more efficient local SCA focus controller. However, as Kuhn et al. 2017a pointed out, spatial

848 aggregation effects have a significant influence on the accuracy of ASI systems.

849 In this work we optimized the controller parameters according to the 7 combined spatial and

850 temporal DNI variability classes. In future works, it could be interesting to investigate the benefit

851 with individually optimized control parameters for each of the 40 theoretically possible

852 combinations of spatial and temporal DNI variability classes.

853 Furthermore, the used ASI system provides also predictions up to 15 minutes ahead. However, 854 currently we do not utilize the predictions for the power plant controller. A further improvement 855 could be achieved by including model predictive control strategies, which could utilize the 856 predictions.

857 The presented new control strategies are tailored to the La Africana solar field design. Other 858 power plants may have fundamentally different control approaches. Yet, to the best of our 859 knowledge the presented class dependent control strategies are the first which use classified 860 spatial DNI information. Spatial DNI information combined with the DNI classification procedure 861 represent additional input information, which could be beneficiary for any existing and future 862 parabolic trough solar field controller, regardless of the used overall control strategy. 
863 Furthermore, we would like to point out that the potential benefit of spatial DNI information

864 combined with variability classification procedures is not limited to parabolic trough power plants.

865 Similar benefits might be feasible for other CSP technologies, such as Fresnel and point

866 focusing tower power plants. Benefits are also expected for solar PV power plants and electrical 867 grids.

868 


\section{Acknowledgment}

870 The European Union funded this research within the H2020 program under the Grant

871 Agreement no. 654984 (PreflexMS). Further funding was received by the German Federal

872 Ministry for Economic Affairs and Energy within the WobaS and WobaS-A projects (Grant

873 Agreement no. 03255848A and 0324307A).

874 Thanks to the colleagues from the Solar Concentrating Systems Unit of CIEMAT for the support 875 provided in the installation and maintenance of the shadow cameras. These instruments are 876 installed on CIEMAT's CESA-I tower of the Plataforma Solar de Almería.

\section{References}

878 Abutayeh, M., Alazzam, A., El-Khasawneh, B., 2014. Balancing heat transfer fluid flow in solar 879 fields, Sol. Energy, 105, 381-389. doi: 10.1016/j.solener.2014.03.025

880 Blanc, P., Massip, P., Kazantzidis, A., Tzoumanikas, P., Kuhn, P., Wilbert, S., Schüler, D., Prahl, C., 2017. Short-Term Forecasting of High Resolution Local DNI Maps with Multiple Fish-Eye Cameras in Stereoscopic Mode, AIP conference Proceedings 1850. doi: 10.1063/1.4984512

883 Burkholder, F., Kutscher, C., 2009. Heat Loss Testing of Schott's 2008 PTR70 Parabolic Trough $884 \quad$ Receiver, Technical Report, NREL.

885 Chow, C.W., Urquhart, B., Lave, M., Dominguez, A., Kleissl, J., Shields, J., Washom, B., 2011. 886 Intra-hour forecasting with a total sky imager at the UC San Diego solar energy testbed. Sol. 887 Energy 85, 2881-2893. doi: 10.1016/j.solener.2011.08. 025. 
888 Crăciun, BI., Kerekes, T., Séra, D., Teodorescu, R., Annakkage, UD.,2017. Power ramp

889 limitation capabilities of large PV power plants with active power reserves. IEEE Trans

$890 \quad$ Sustainable Energy, 8, 573-581. doi: 10.1109/tste.2016.2612121

891 Denholm, P., Margolis, R., 2016. Energy Storage Requirements for Achieving 50\% Solar 892 Photovoltaic Energy Penetration in California, NREL/TP-6A20-66595, doi: 10.2172/1298934

893 Dersch, J., Hennecke, K., Quaschning, V.. 2012. Free Greenius - New Options and Availability. 894 In Proceedings of the SolarPACES 2012 Conference, Marrakesch (Marokko)

895 Dieckmann, S., 2017. DLR - Institut of Solar Research - greenius - The green energy system 896 analysis tool. URL http://www.dlr.de/sf/desktopdefault.aspx/tabid-11688/20442_read-44865/

897 García, I., Álvarez, J., Blanco, D., 2011. Performance model for parabolic trough solar thermal 898 power plants with thermal storage: comparison to operating plant data. Sol. Energy 95, 899 2443-2460. doi: 10.1016/j.solener.2011.07.002

900 Gevorgian, V., Booth, S., Review of PREPA technical requirements for interconnecting wind and 901 solar generation, NREL/TP; 5 D 00- 57089, 2013, available online:

$902 \quad$ http://www.nrel.gov/docs/fy14osti/57089.pdf

903 Giostri, A., 2012.Transient effects in linear concentrating solar thermal power plant, Ph.D. thesis, 904 Energy Department, Politecnico Di Milano http://hdl.handle.net/10589/89590

905 Hanrieder, N., Sengupta, M., Xie, Y., Wilber, S., Pitz-Paal, R., 2016. Modeling beam attenuation 906 in solar tower plants using common DNI measurements. Solar Energy 129, pp. 244-255. doi: $907 \quad$ 10.1016/j.solener.2016.01.051 
908 Hirsch, T., Schenk, HE., 2010. Dynamics of oil-based parabolic trough plants—A detailed 909 transient simulation model. In Proceedings of the SolarPACES 2010 Conference, Perpignan $910 \quad$ (France)

911 Hirsch, T., Martin, N., Gonzalez. L., Biencinto, M., Wilbert, S., Schroedter-Homscheidt, M., 912 Chenlo, F., Feldhoff, J., 2014. Direct Normal Irradiance Nowcasting methods for optimized 913 operation of concentrating solar technologies, DNICast project, DNIcast Deliverable 2.1. 914 http://www.dnicast-project.net.

915 IRENA, 2019. Renewable capacity statistics 2019, International Renewable Energy Agency 916 (IRENA), Abu Dhabi. ISBN 978-92-9260-123-2

917 Janotte, N., 2012. Requirements for Representative Acceptance Tests for the Prediction of the 918 Annual Yield of Parabolic Trough Solar Fields, Ph.D. thesis, Fakultät für Maschinenwesen 919 der Rheinisch-Westfälischen Technischen Hochschule Aachen, Aachen Germany

920 Kazantzidis, A., Tzoumanikas, P., Blanc, P., Massip, P., Wilbert, S., Ramirez-Santigosa, L., 921 2017. Short-term forecasting based on all-sky cameras, In: Renewable Energy Forecasting. 922 Woodhead Publishing Series in Energy. Woodhead Publishing, pp. 153-178. doi: 923 10.1016/B978-0-08-100504-0. 00005-6.

924 Kost, C., Shammugam, S., Jülch, V., Nguyen, H., Schlegl, T., 2018. Levelized cost of electricity 925 renewable energy technologies, Fraunhofer ISE,

926 https://www.ise.fraunhofer.de/en/publications/studies/cost-of-electricity.html

927 Kuhn, P., Nouri, B., Wilbert, S., Prahl, C., Kozonek, N., Schmidt, T., Yasser, Z., Ramirez, L., 928 Zarzalejo, L., Meyer, A., Vuilleumier, L., Heinemann, D., Blanc, P., Pitz-Paal, R., $2017 a$. 
929 Validation of an all-sky imager-based nowcasting system for industrial PV plants. Prog.

930 Photovolt.: Res. Appl. doi: 10.1002/pip.2968.

931 Kuhn, P., Wilbert, S., Prahl, C., Schüler, D., Haase, T., Hirsch, T., Wittmann, M., Ramirez, L., 932 Zarzalejo, L., Meyer, A. and Vuilleumier, L., 2017b. Shadow camera system for the 933 generation of solar irradiance maps. Sol. Energy 157, 157-170. doi:

$934 \quad$ 10.1016/j.solener.2017.05.074

935 Kuhn, P., Garsche, D., Wilbert, S., Nouri, B., Hanrieder, N., Prahl, C., Zarzalejo, L., Fernández, 936 J., Kazantzidis, A., Schmidt, T., Heinemann, D., Blanc, P., Pitz-Paal, R., 2019, Shadow937 camera based solar nowcasting system for shortest-term forecasts. Meteorologische $938 \quad$ Zeitschrift (submitted)

939 Lilliestam, J., Barradi, T., Caldés, N., Gomez, M., Hanger, S., Kern, J., Komendantova, N., 940 Mehos, M., Hong, WM., Wang, Z., Patt, A., 2018. Policies to keep and expand the option of 941 concentrating solar power for dispatchable renewable electricity. Energy policy, 116, 193942 197. doi: 10.1016/j.enpol.2018.02.014

943 Lilliestam, J., Pitz-Paal, R., 2018. Concentrating solar power for less than USD 0.07 per kWh: 944 finally the breakthrough?. Renewable Energy Focus, 26:17-21. doi:

$945 \quad$ 10.1016/j.ref.2018.06.002

946 Marquez, R., Coimbra, C.F., 2013. Proposed metric for evaluation of solar forecasting models. J. 947 Sol. Energy Eng. 135 (1). doi: org/10.1115/1.4007496.

948 Mehos, M., Turchi, C., Jorgenson, J., Denholm, P., Ho, C., Armijo, K., 2016. On the Path to 949 SunShot: Advancing Concentrating Solar Power Technology, Performance, and 
Dispatchability. Golden, CO: National Renewable Energy Laboratory. NREL/TP-5500-65688. http://www.nrel.gov/docs/fy16osti/65688.pdf.

952 Noureldin, K., González-Escalada, L.M., Hirsch, T., Nouri, B., Pitz-Paal, R., 2016. Modelling and 953 optimization of transient processes in line focusing power plants with single-phase heat 954 transfer medium, AIP Conference Proceedings. Vol. 1734. doi: 10.1063/1.4949169

955 Noureldin, K., Hirsch, T., Pitz-Paal, R., 2017. Virtual Solar Field-Validation of a detailed transient 956 simulation tool for line focus STE fields with single phase heat transfer fluid. Sol. Energy146, 957 131-140. doi: 10.1016/j.solener.2017.02.028

958 Noureldin, K., Hirsch, T., Nouri, B., Yasser, Z., 2018a. Simulation of Potential Enhancements in 959 Parabolic Trough Solar Field Start-up Controllers using Nowcasting Systems. 24th 960 SolarPACES Conference (Casablanca, Morocco)

961 Noureldin, K., Hirsch, T., Kuhn, P., Nouri, B., Yasser, Z., Pitz-Paal, R., 2018b. Modelling an 962 automatic controller for parabolic trough solar fields under realistic weather conditions. AIP 963 Conference Proceedings. Vol. 2033. doi: 10.1063/1.5067211

964 Noureldina, K., Hirscha, T., Nouri, B., Yasserc, Z., Pitz-Paald, R., 2019a. Evaluating the 965 potential benefit of using nowcasting systems to improve the yield of parabolic trough power 966 plants with single-phase HTF. Applied energy (submitted)

967 Noureldin, K., 2019b. Modelling and Optimization of Transient Processes in Parabolic Trough 968 Power Plants with Single-Phase Heat Transfer Fluid. PhD Dissertation, RWTH Aachen, 969 Aachen (Germany) 
970 Nouri, B., Röger, M., Janotte, N., Hilgert, C., 2018a Characterization and corrections for clamp971 on fluid temperature measurements in turbulent flows. Journal of Thermal Science and $972 \quad$ Engineering Applications. Vol. 10, 031011.

973 Nouri, B., Kuhn, P., Wilbert, S., Prahl, C., Pitz-Paal, R., Blanc, P., Schmidt, T., Yasser, Z., 974 Ramirez Santigosa, L., Heineman, D., 2018b. Nowcasting of DNI Maps for the Solar Field 975 Based on Voxel Carving and Individual 3D Cloud Objects from All Sky Images, AIP 976 Conference Proceedings. Vol. 2033. doi:10.1063/1.5067196

977 Nouri, B., Kuhn, P., Wilbert, S., Hanrieder, N., Prahl C., Zarzalejo, L., Kazantzidis, A., Blanc, P., 978 Pitz-Paal, R., 2019a, Cloud height and tracking accuracy of three all sky imager systems for 979 individual clouds. Sol. Energy 177, 213-228. doi: 10.1016/j.solener.2018.10.079

980 Nouri, B., Wilbert, S., Segura, L., Kuhn, P., Hanrieder, N., Kazantzidis, A., Schmidt, T., 981 Zarzalejo, L., Blanc, P., Pitz-Paal, R.,2019b, Determination of cloud transmittance for all sky 982 imager based solar nowcasting. Sol. Energy, 181, 251-263. doi:

$983 \quad$ 10.1016/j.solener.2019.02.004.

984 Nouri, B., Wilbert, S., Kuhn, P., Hanrieder, N., Schroedter-Homscheidt, M., Kazantzidis, A., 985 Zarzalejo, L., Blanc, P., Kumar, S., Goswami, N., Shankar, R., Affolter, R., Pitz-Paal, R., 986 2019c. Real-Time Uncertainty Specification of all Sky Imager Derived Irradiance Nowcasts. 987 Remote Sens. 11(9), 1059. doi: 10.3390/rs11091059

988 Peng, Z., Yu, D., Huang, D., Heiser, J., Yoo, S., Kalb, P., 2015. 3D cloud detection and tracking 989 system for solar forecast using multiple sky imagers Sol. Energy 118, 496-519. 990 https://doi.org/10.1016/j.solener.2015.05.037. 
991 Perez, R., David, M., Hoff, T.E., Jamaly, M., Kivalov, S., Kleissl, J., Lauret, P., Perez, M., 2016. 992 Spatial and temporal variability of solar energy. Found. Trends Renew. Energy 1 (1), 1-44. 993 doi: $10.1561 / 2700000006$.

994 Pitz-Paal, R., 2017. Concentrating solar power: still small but learning fast. Nature Energy. 995 2(7):17095. doi: 10.1038/nenergy.2017.95

996 Platzer, WJ., 2016. Combined solar thermal and photovoltaic power plants-An approach to 24h 997 solar electricity?. AIP Conference Proceedings. Vol. 1734. doi: 10.1063/1.4949173

998 Quesada-Ruiz, S., Chu, Y., Tovar-Pescador, J., Pedro, H., Coimbra, C., 2014. Cloud tracking 999 methodology for intra-hour DNI forecasting. Sol. Energy 102, 267-275. doi: $1000 \quad$ 10.1016/j.solener.2014.01.030.

1001 Schlichting, T., 2018. Bewertung der Verwendbarkeit von Strahlungskarten für den Einsatz in 1002 der Regelung eines Parabolrinnensystems, Master thesis, Fakultät für 1003 Ingenieurwissenschaften, Universität Duisburg-Essen, Germany

1004 Schroedter-Homscheidt, M., Kosmale, M., Jung, S., Kleissl, J., 2018. Classifying ground1005 measured 1 minute temporal variability within hourly intervals for direct normal irradiances. $1006 \quad$ Meteorol. Z. 2018. doi: 10.1127/metz/2018/0875.

1007 Wagner, PH., Wittmann, M., 2014. Influence of different operation strategies on transient solar 1008 thermal power plant simulation models with molten salt as heat transfer fluid. Energy 1009 Procedia, 49, 1652-1663. doi: 10.1016/j.egypro.2014.03.174

1010 Wilbert, S., Nouri, B., Prahl, C., Garcia, G., Ramirez, L., Zarzalejo, L., Valenzuela, L., Ferrera, 1011 F., Kozonek, N., Liria, J., 2016. Application of Whole Sky Imagers for Data Selection for 
1012 Radiometer Calibration. In: EU PVSEC 2016 Proceedings, 1493-1498. doi:

1013 10.4229/EUPVSEC20162016-5AO.8.6

1014 Woyte, A., Van Thong, V., Belmans, R., Nijs, J., 2006. Voltage fluctuations on distribution level 1015 introduced by photovoltaic systems. IEEE Transactions on energy conversion, 21, 202-209. 1016 doi: $10.1109 /$ TEC.2005.845454

1017 Zaversky, F., Medina, R., García-Barberena, J., Sánchez, M., Astrain, D., 2013. Object-oriented 1018 modeling for the transient performance simulation of parabolic trough collectors using molten 1019 salt as heat transfer fluid. Sol. Energy, 95, 192-215. doi: 10.1016/j.solener.2013.05.015 